\title{
The crossover function of MutS $\gamma$ is activated via Cdc7-dependent stabilization of Msh4
}

Authors: Wei He ${ }^{1,2}$, H.B.D. Prasada Rao ${ }^{1,2, \dagger}$, Shangming Tang ${ }^{1,2}$, Nikhil Bhagwat ${ }^{1,2}$,

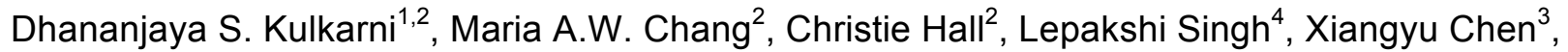
Nancy M. Hollingsworth ${ }^{3}$, Petr Cejka ${ }^{4}$ and Neil Hunter ${ }^{1,2,5,6}$

\section{Affiliations:}

${ }^{1}$ Howard Hughes Medical Institute, University of California, Davis, Davis, California, USA.

${ }^{2}$ Department of Microbiology \& Molecular Genetics, University of California, Davis, Davis, California, USA.

${ }^{3}$ Department of Biochemistry and Cell Biology, Stony Brook University, Stony Brook, New York, U.S.A.

${ }^{4}$ Institute for Research in Biomedicine, Università della Svizzera italiana, Bellinzona, Switzerland.

${ }^{5}$ Department of Molecular \& Cellular Biology, University of California, Davis, Davis, California, USA.

${ }^{6}$ Department of Cell Biology \& Human Anatomy, University of California, Davis, Davis, California, USA.

† Current address: National Institute of Animal Biotechnology, Hyderabad, Telangana, India.

*Correspondence to: Neil Hunter, E-mail: nhunter@ucdavis.edu 


\section{SUMMARY}

The MutS $\gamma$ complex, Msh4-Msh5, binds DNA joint-molecule (JM) intermediates during homologous recombination to promote crossing over and accurate chromosome segregation at the first division of meiosis. MutS $\gamma$ facilitates the formation and biased resolution of crossoverspecific JM intermediates called double Holliday junctions. Here we show that these activities are governed by regulated proteasomal degradation. MutS $\gamma$ is initially inactive for crossing over due to an N-terminal degron on Msh4 that renders it unstable. Activation of MutS $\gamma$ requires the Dbf4-dependent kinase, Cdc7 (DDK), which directly phosphorylates and thereby neutralizes the Msh4 degron. Phosphorylated Msh4 is chromatin bound and requires DNA strand exchange and chromosome synapsis, implying that DDK specifically targets MutS $\gamma$ that has already bound nascent JMs. Our study establishes regulated protein degradation as a fundamental mechanism underlying meiotic crossover control. 


\section{INTRODUCTION}

Crossing over is required for the accurate segregation of homologous chromosomes (homologs) at the first division of meiosis (Hunter, 2015; Watanabe, 2012). At metaphase I, the four chromatids of a bivalent are interconnected by cohesion between sister chromatids and at least one crossover between the homologs. These connections enable stable bipolar attachment of bivalents to the meiosis-I spindle, which is a prerequisite for accurate homolog disjunction at the ensuing anaphase. Crossing over is highly regulated to ensure that homolog pairs obtain the requisite connection despite a low average number of crossovers per nucleus (Jones, 1984). Also, crossovers between a single homolog pair inhibit one another such that multiple events tend to be widely and evenly spaced (Wang et al., 2015). Together, these crossover assurance and crossover interference processes dictate the range of crossover numbers per nucleus. In $C$. elegans, interference is effective along the entire lengths of all chromosomes, minimizing crossover numbers to one per chromosome (Hillers and Villeneuve, 2003). More typically, crossovers average around one per chromosome arm, but smaller chromosomes will often obtain only a single exchange. Suboptimal crossing-over, leading to missegregation and aneuploidy, and aberrant exchange between non-allelic homologies are leading causes of congenital disease in humans (Herbert et al., 2015; Hunter, 2015; Kim et al., 2016; Wang et al., 2017).

The regulatory processes that underlie crossover control remain poorly understood. Crossover sites are designated from a larger pool of recombination sites initiated by Spo11-catalyzed DNA double-strand breaks (DSBs)(Lam and Keeney, 2014). DSBs outnumber crossovers by 2-fold in budding yeast, $\sim 10$-fold in mammals and up to 30 -fold in some plants. At the cytological level, the differentiation of crossover sites manifests as the selective retention and accumulation of specific recombination factors. One such factor is MutS $\gamma$, a heterodimer of Msh4 and Msh5, two homologs of the DNA mismatch-recognition factor MutS (Manhart and Alani, 2016; Pochart et 
al., 1997; Snowden et al., 2004). Msh4 and Msh5 are members of the ZMM proteins (Zip1, Zip2, Zip3, Zip4, Msh4, Msh5, Mer3, and Spo16), a diverse set of factors that facilitate crossoverspecific recombination events, and couple these events to chromosome synapsis (Borner et al., 2004; Fung et al., 2004; Hunter, 2015; Lynn et al., 2007; Shinohara et al., 2008). As seen in a variety of species, initial numbers of MutS $\gamma$ immunostaining foci greatly outnumber final crossover numbers (De Muyt et al., 2014; de Vries et al., 1999; Edelmann et al., 1999; Higgins et al., 2008; Kneitz et al., 2000; Yokoo et al., 2012; Zhang et al., 2014). As prophase I progresses, MutS $\gamma$ is lost from most recombination sites but retained at sites that mature into crossovers. This patterning process is dependent on the Zip3/RNF212/ZHP-3/HEI10 family of RING E3 ligases (Agarwal and Roeder, 2000; Bhalla et al., 2008; Chelysheva et al., 2012; De Muyt et al., 2014; Henderson and Keeney, 2004; Jantsch et al., 2004; Qiao et al., 2014; Rao et al., 2017; Reynolds et al., 2013; Wang et al., 2012; Yokoo et al., 2012; Zhang et al., 2018). Additional evidence implicates the SUMO-modification and ubiquitin-proteasome systems in meiotic crossover control (Ahuja et al., 2017; Rao et al., 2017) and suggests a model in which factors such as MutS $\gamma$ are selectively stabilized at crossover sites by protecting them from proteolysis. Implicit in this model is the notion that MutS $\gamma$ is intrinsically unstable.

Here, we show that regulated proteolysis plays a direct and essential role in meiotic crossing over. Msh4 is identified as an intrinsically unstable protein that is targeted for proteasomal degradation by an N-terminal degron thereby rendering MutS $\gamma$ inactive for crossing over. Activation of MutS $\gamma$ occurs by neutralizing the Msh4 degron via phosphorylation catalyzed by the conserved cell-cycle kinase, Cdc7-Dbf4 (DDK). Thus, a key meiotic pro-crossover factor is activated by attenuating its proteolysis. 


\section{RESULTS}

\section{The N-terminal Region of Msh4 is Phosphorylated}

The ZMM proteins were surveyed for modifications detectable as electrophoretic-mobility shifts on Western blots. A prominent modified band was detected for Msh4 but not for its partner Msh5 (Figure 1A). Treatment of immunoprecipitated Msh4 with $\lambda$ phosphatase indicated that the modified form is due to phosphorylation (Figure 1B). Relative to the unphosphorylated protein, phosphorylated Msh4 appeared with a $\geq 1 \mathrm{hr}$ delay, its levels peaked at $\sim 22 \%$ of total protein, and then both species disappeared with the same timing (Figure 1C). To map sites of phosphorylation, Msh4 was immunoprecipitated, fast and slow migrating forms were resolved by electrophoresis, and then analyzed separately by tandem mass spectrometry (MS/MS; Figure 1D). Six phosphorylation sites were identified in the slower migrating form of Msh4, all mapping within the first 50 amino acids (S2, S4, S7, S41, T43 and S46; Figure 1E; Supplemental

Figure S1). In the faster migrating form of Msh4, only phosphorylation at S41 was detected.

Msh4 and Msh5 lack the N-terminal domain I, which is conserved in other MutS proteins

(Figure 1E). Domain I encircles DNA together with MutS domain IV and is intimately involved in DNA binding and mismatch recognition (Yang et al., 2000). Absence of domain I from Msh4 and Msh5 is predicted to enlarge the DNA channel such that it can accommodate JM structures and slide on two duplexes (Rakshambikai et al., 2013; Snowden et al., 2004). The functions of the $\mathrm{N}$-terminal regions of Msh4 and Msh5 are otherwise unknown.

\section{Phosphorylation is Essential for the Crossover Function of Msh4}

To determine the functional relevance of Msh4 phosphorylation, we mutated the six identified phosphorylation sites to alanine to prevent phosphorylation, or to aspartic acid to mimic phosphorylation, creating respectively $m s h 4-6 A$ and $m s h 4-6 D$ alleles (Figure 2). Spore viability, 
indicative of successful meiotic chromosome segregation, was assessed by tetrad dissection and compared to wild-type and msh4 $\Delta$-null mutant strains (Figure 2A,B). Consistent with previous studies (Krishnaprasad et al., 2015; Nishant et al., 2010; Novak et al., 2001; Stahl et al., 2004), $m s h 4 \Delta$ reduced spore viability to $34.7 \%$ and the pattern of spore death was indicative of chromosome missegregation at the first meiotic division, with a preponderance of tetrads containing two or zero viable spores (Figure 2B). The pattern of spore death in cells carrying the phosphorylation-defective $m s h 4-6 A$ allele was similar to that of the $m s h 4 \Delta$ null, with an overall viability of $46.7 \%$ ( $P<0.01$ compared to wild type, $\chi^{2}$ test; Table S1). By contrast, the phospho-mimetic msh4-6D allele supported wild-type levels of spore viability $(96.3 \%$ and $95.7 \%$, respectively, $P=0.42)$.

In the absence of Msh4, chromosome missegregation and the ensuing spore death are caused by defective crossing over (Krishnaprasad et al., 2015; Nishant et al., 2010; Novak et al., 2001; Stahl et al., 2004). To assess whether phosphorylation is required for the crossover function of Msh4, we measured genetic map distances in a background carrying markers on three different chromosomes (III, VII and VIII; Figure 2C). Cumulative map distances showed that $m s h 4 \Delta$ reduced crossing by 2.1 to 2.7-fold (Figure 2D and Supplemental Figure S2 and Table S2). Similar reductions (2.1 to 2.8 -fold) were seen for the $m s h 4-6 A$ phosphorylation-defective strain. Thus, phosphorylation is essential for the crossover function of Msh4. For chromosomes VII and VIII, the msh4-6A mutation caused slightly larger reductions in crossing over than the $m s h 4 \Delta$ null. Possibly, phosphorylation-defective Msh4-6A protein is still capable of binding recombination intermediates thereby impeding processing via alternative crossover pathways mediated by the structure-selective nucleases (De Muyt et al., 2012; Zakharyevich et al., 2012). Consistent with the high spore viability of the phospho-mimetic msh4-6D strain, cumulative map distances for this strain were indistinguishable from those of wild type. 
Previous analysis showed that non-crossover gene conversions are increased in the absence of ZMMs, including Msh5 (and by extension Msh4), due to the continued formation of DSBs when homolog engagement is defective (Hollingsworth et al., 1995; Novak et al., 2001; Thacker et al., 2014). This phenotype was reflected in tetrad data from the $m s h 4 \Delta$ null strain, which showed a 3.4-fold increase in the cumulative gene conversion frequency for the 12 markers in this background (Figure 2E; also see Supplemental Figure S2). Elevated gene conversion was also seen for $m s h 4-6 A$, which showed a 2.5 -fold increase in gene conversions relative to wild type. Unexpectedly, a 1.7-fold increase in gene conversion was observed for the $m s h 4-6 D$ strain, the first indication that this phospho-mimetic allele does not possess fully wild-type function.

Crossovers promoted by MutS $\gamma$ are patterned by interference (Krishnaprasad et al., 2015; Novak et al., 2001; Stahl et al., 2004). One readout of crossover interference is that tetrads with crossovers in a given "test" interval have significantly lower frequencies of crossovers in the neighboring intervals when compared to tetrads that lack crossovers in the test interval (Malkova et al., 2004)(Figure 2F). This difference can be expressed as the ratio of map distances for the neighboring interval in the tetrad subsets with or without a crossover in the test interval. Positive crossover interference is indicated by a ratio that is significantly less than one. To address whether Msh4 phosphorylation promotes crossovers with an interference distribution, this analysis was performed for all interval pairs (Figure 2F; also see

\section{Supplemental Figure S3).}

In wild-type tetrads, significant positive crossover interference was detected for all interval pairs except LEU2-CEN3-MAT on chromosome 3 (Figure 2F). Consistent with previous studies

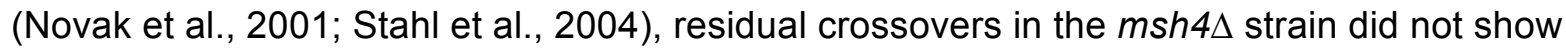
significant positive interference in any interval pair. In fact, significant negative interference - a 
higher incidence of double crossovers than expected - was detected for one interval pair on chromosome VII (LYS5-MET13-CYH2) in msh4D tetrads. Analogous results were obtained for the msh4-6A strain indicating that Msh4 phosphorylation does indeed promote the formation of crossovers that are subject to interference. However, significant positive interference was still detected in one interval pair for msh4-6A (ARG4-THR1-CUP1). By contrast, crossover interference in the msh4-6D strain was similar to wild type, with the exception of one interval pair in which interference was diminished (LYS5-MET13-CYH2; Figure 2F). These exceptions suggest that the msh4-6A strain may not be completely defective for the formation of interfering crossovers, while msh4-6D may not be fully competent for this function.

Interference within individual intervals was also analyzed by calculating non-parental ditype (NPD) tetrad ratios (Figure 2G and Supplemental Table S4). Within a given interval, a double crossovers event involving all four chromatids results in a NPD tetrad. The NPD ratio compares the number of NPDs observed to that expected if there were no crossover interference (Papazian, 1952). A ratio of significantly less than one indicates positive crossover interference. Residual crossovers in the $m s h 4 \Delta$ and $m s h 4-6 A$ strains did not show significant interference by NPD analysis. In the msh4-6D strain, interference was detected in all but one interval (CEN3$M A T)$. However, in wild-type tetrads, significant interference was detected in the same interval, again suggesting that $m s h 4-6 D$ is slightly defective for crossover interference

Next, we determined whether crossover assurance is influenced by Msh4 phosphorylation using a strain carrying eight linked intervals that span the length of chromosome III (Figure 2H)(Zakharyevich et al., 2010). In wild type, at least one crossover was detected in $98.9 \%$ of tetrads indicating highly efficient crossover assurance (Figure 2I). Oppositely, crossover assurance was severely defective in the absence of Msh4, with $25.2 \%$ of $m s h 4 \Delta$ tetrads lacking a detectable crossover on chromosome III, consistent with previous analysis (Krishnaprasad et 
al., 2015). In addition, the fraction of tetrads with a single crossover was increased and multiple crossover classes were diminished in the $m s h 4 \Delta$ strain relative to wild type $(P<0.001, G$-test $)$. If crossover assurance remained operational in msh4-6A cells, the residual crossover frequency along chromosome III (1.7 crossovers per meiosis) is in principle sufficient to ensure a crossover in every meiosis. Contrary to this scenario, $17.4 \%$ of $m s h 4-6 A$ tetrads had zero crossovers highlighting the importance of Msh4 phosphorylation for crossover assurance (Figure 2l; $P<0.001$ compared to wild type, G-test; distributions of crossover classes were not different for $m s h 4-6 A$ and $m s h 4 \Delta, P=0.38)$. The phospho-mimetic $m s h 4-6 D$ strain was not significantly different from wild type for crossover assurance, with just $2.8 \%$ of tetrads lacking a crossover $(P=0.79)$. In conclusion, phosphorylation of the $\mathrm{N}$-terminus of Msh4 promotes the formation of crossovers that are subject to patterning processes that result in crossover assurance and interference.

The contributions of individual phosphorylation sites to the crossover function of Msh4 were also assessed. This analysis revealed a major role for phosphorylation at sites S2, S4 and S7, while S41, T43 and S46 made little or no contribution to crossing over (Supplemental Figure S3). Western analysis indicated that Msh4-6A protein could still be phosphorylated, albeit with a delay and at lower levels than wild-type Msh4 (Supplementary Figure S5; low-level phosphorylation was also detected for Msh4-6D; also see Figure 5A;). This residual phosphorylation was abolished following mutation of all 18 serine and threonine residues present in the first 50 amino acids of Msh4 indicating that phosphorylation leading to the slow migrating form is confined to this region (Supplementary Figure S5). Importantly, the msh4$18 \mathrm{~A}$ strain was no more defective for crossing over than the msh4-6A strain, indicating that phosphorylation of other sites in the $\mathrm{N}$-terminus is not functionally redundant with the phosphorylation sites mapped by MS/MS (Supplementary Figure S3). 


\section{Msh4 Phosphorylation Facilitates the Formation and Resolution of DNA Joint Molecules}

To understand how the molecular steps of meiotic recombination are influenced by Msh4 phosphorylation, DNA intermediates were monitored in cultures undergoing synchronous meiosis using a series of Southern blot assays at the well-characterized HIS4::LEU2 recombination hotspot (Hunter and Kleckner, 2001; Oh et al., 2007). At this locus, Xhol polymorphisms between the two parental chromosomes produce DNA fragments diagnostic for DSBs, JMs, and crossover products. DSBs, and crossovers were analyzed using onedimensional (1D) gels (Figure 3A-C). Noncrossover products were detected by monitoring conversion of a BamHI/NgoMIV restriction-site polymorphism located directly at the site of DSB formation (Figure 3A and 3D).

Analysis of JM intermediates in budding yeast together with fine-scale analysis of recombination products in a variety of species, indicate that crossover and noncrossover pathways diverge at an early step, following nascent D-loop formation (Allers and Lichten, 2001b; Borner et al., 2004; Drouaud et al., 2013; Guillon et al., 2005; Hunter and Kleckner, 2001; Jeffreys and May, 2004; Marsolier-Kergoat et al., 2018; Martini et al., 2011; Rockmill et al., 2013; Wijnker et al., 2013). A majority of noncrossovers arise from D-loops via synthesis-dependent strand annealing in which the invading 3' end is extended by DNA polymerase, unwound and then annealed to the other DSB end (Marsolier-Kergoat et al., 2018; McMahill et al., 2007). By contrast, most crossovers form via metastable one-ended strand-exchange intermediates called single-end invasions (SEIs), which form as homologs synapse (Hunter and Kleckner, 2001). Through DNA synthesis and second-end capture, SEls give rise to double-Holliday junctions (dHJs)(Lao et al., 2008), which must then undergo biased resolution into crossovers (Zakharyevich et al., 2012). Native/native two-dimensional (2D) gels reveal the branched structure of JMs and were used to quantify SEls, inter-homolog $\mathrm{dHJs}(\mathrm{IH}-\mathrm{dHJs})$, inter-sister JMs (IS-JMs) and multi-chromatid JMs (mc-JMs) comprising three and four interconnected DNA 
molecules (Figure 3E and 3F). To monitor the timing and efficiency of meiotic divisions, fixed cells were stained with DAPI and scored as having one, two, or four nuclei.

In wild-type, $m s h 4 \Delta, m s h 4-6 A$ and $m s h 4-6 D$ strains, DSBs appeared and reached peak levels with similar timing (Figure 3C). Peak DSB levels were higher and DSBs disappeared with a $\sim 1$ $\mathrm{hr}$ delay in the $m s h 4 \Delta$ null mutant relative to wild type, consistent with delayed progression of recombination and continued DSB formation (Borner et al., 2004; Thacker et al., 2014). Delayed progression in $m s h 4 \Delta$ cells was also reflected by a $\sim 2 \mathrm{hr}$ delay of the meiosis-I division (MI)

(Figure 3C). Mirroring the crossover reductions detected by tetrad analysis, crossovers at HIS4::LEU2 were reduced $\sim 2$-fold in $m s h 4 \Delta$ cells. A similar reduction in crossing over was seen for $m s h 4-6 A$ cells, but progression defects were less severe than those seen for $m s h 4 \Delta$; disappearance of DSBs was delayed by $\sim 30$ minutes and MI was delayed by $\sim 1.25 \mathrm{hrs}$. In msh4-6D cells, slight delays ( $\leq 20$ minutes) in DSB turnover and crossover formation were apparent, but crossovers reached wild-type levels.

The increased frequencies of gene conversion seen in $m s h 4 \Delta$ and $m s h 4-6 A$ tetrads (Figure 2E) were mirrored by elevated levels of non-crossover gene conversions at HIS4::LEU2 (Figure 3D). Again, the effect of $m s h 4-6 A$ was weaker than that of the $m s h 4 \Delta$ null (increases of 1.8-fold versus 3.0-fold, respectively). Although gene conversion was also significantly elevated in msh4-6D tetrads, non-crossovers at HIS4::LEU2 were not significantly increased.

Two-dimensional gel analysis revealed the importance of Msh4 phosphorylation for JM metabolism (Figure 3E-H). In msh4-6A cells, appearance of all JM species was delayed relative to wild-type by $\sim 30-60$ min (Figure $3 \mathbf{G}$ ), with the longest delays seen for JMs involving strand-exchange between sister chromatids, i.e. IS-JMs and mc-JMs. A further delay of $\sim 1.5$ hrs was seen for the disappearance of JMs in $m s h 4-6 A$ relative to wild type. Peak JM levels were 
also lower in msh4-6A cells, averaging $61 \%$ of wild-type levels (Figure $3 \mathbf{H}$ ). JM kinetics in msh4 4 null mutants were similar to those of msh4-6A (Figure $3 \mathbf{G}$ ), but peak JM levels were significantly lower averaging just $40 \%$ of wild-type levels (Figure $3 \mathrm{H}$ ). Thus, with respect to DSB persistence, JM levels and prophase delay, the phenotypes of the msh4-6A mutant are milder than those of the $m s h 4 \Delta$ null, but still severely defective relative to wild type or $m s h 46 D$ strains.

Notably, SEls reached similar levels in $m s h 4-6 A$ and $m s h 4 \Delta$ cells $(\%$ of hybridizing DNA $=$ $1.19 \% \pm 0.05$ S.E. and $0.91 \% \pm 0.13$ S.E., respectively), but $\mathrm{dHJ}$ levels were $\sim 2$-fold lower in $m s h 4 \Delta$ cells $(0.83 \% \pm 0.05$ S.E. and $0.40 \% \pm 0.02$ S.E., respectively). Two non-exclusive possibilities could explain this difference: (i) the SEl-to-dHJ transition is less efficient and/or (ii) the stability of $\mathrm{IH}-\mathrm{dHJ}$ is lower in the absence of Msh4 than when the Msh4-6A protein is present. However, despite higher IH-dHJ levels in $m s h 4-6 A$ cells, final crossover levels were very similar to those of the msh4 $\Delta$ null (Figures 2D, 2H and $3 \mathbf{C}$ ). Together, these data suggest that phosphorylation of Msh4 is important both for JM formation and the crossover-biased resolution of $\mathrm{IH}-\mathrm{dHJs}$.

In msh4-6D cells, a minor delay in SEl formation was observed and $\mathrm{IH}-\mathrm{dHJ}$ seaked at $\sim 24 \%$ higher levels relative to wild type $(1.58 \% \pm 0.11$ versus $1.27 \% \pm 0.11)$. But overall, JM kinetics and levels in msh4-6D cells were similar to those of wild type (Figure $\mathbf{3 G}$ and $\mathbf{3 H}$ ).

\section{Msh4 Phosphorylation Facilitates Homolog Synapsis}

In most organisms, the strand-exchange step of meiotic recombination promotes the pairing of homologs and their intimate end-to-end connection by synaptonemal complexes (SCs), meiosisspecific structures comprising densely-packed transverse filaments (Zickler and Kleckner, 2015). Crossovers then mature in the context of SCs, which are subsequently disassembled leaving homologs connected only at the sites of exchange. The possibility that the delayed and 
inefficient JM formation seen in msh4-6A cells leads to defective homolog synapsis was addressed by immuno-staining surface-spread nuclei for the synaptonemal complex transversefilament protein, Zip1 (Figure 4A and 4B)(Dong and Roeder, 2000). Synapsis was monitored over time by assigning nuclei to one of three classes based on the pattern of Zip1 staining (Borner et al., 2004): class I nuclei were defined by a dotty pattern; class II nuclei had partial synapsis with both linear and dotty staining; and class III had full synapsis indicated by extensive linear staining. Nuclei containing aggregates of Zip1 called polycomplexes (PCs), a sensitive indicator of synapsis defects (Sym and Roeder, 1995), were also quantified irrespective of their staining class.

In wild-type cells, peak levels of class III nuclei (35\%) with full synapsis were seen at $5 \mathrm{hrs}$, and Zip1 has disappeared by 8 hrs (Figure 4B). Consistent with previous studies (Borner et al., 2004; Novak et al., 2001), synapsis was severely defective in $m s h 4 \Delta$ null cells, with only $9 \%$ of cells achieving full synapsis and PCs present in a majority of cells (Figure 4B). PCs were similarly prominent in msh4-6A cells, but synapsis was slightly more efficient, with significantly higher levels of class II and class III nuclei $(P<0.005$, G-test). By contrast, synapsis in msh4-6D cells was indistinguishable from wild-type cells $(P=0.63)$. Thus, Msh4 phosphorylation facilitates the formation and/or stabilization of SCs.

\section{Phosphorylation Promotes Chromosomal Localization of Msh4}

To begin to understand how phosphorylation facilitates Msh4 function, the chromosomal localization patterns of Msh4, Msh4-6A and Msh4-6D proteins were compared. Surface spread nuclei were immunostained for both Msh4 and Zip1 (Figure 4B and 4C). Msh4 foci were quantified in nuclei with zygotene (class II) and pachytene (class III) morphologies, i.e. partial and complete lines of Zip1 staining. In wild-type, Msh4 foci averaged $43.9 \pm$ 13.3 S.D. per nucleus while focus numbers in $m s h 4-6 A$ nuclei were lower, averaging $33.8 \pm 10.4$ S.D. 
$(P<0.0001$, two-tailed Mann Whitney test; Figure 4C). By contrast, the Msh4-6D protein formed slightly elevated numbers of foci relative to wild-type Msh4, averaging $47.7 \pm 11.3$ S.D. per nucleus $(P=0.028)$. Phenotypes associated with phosphorylation-defective $(m s h 4-3 A)$ and phosphorylation-mimetic ( $m s h 4-3 D$ ) alleles for sites S2, S4 and S7 were analogous to those of msh4-6A and msh4-6D with respect to formation of Msh4 foci (Supplementary Figure S4), further highlighting the importance of these three proximal serine residues.

\section{Msh4 is Stabilized by Phosphorylation}

We explored the possibility that aberrant localization of phosphorylation-defective Msh4-6A protein is caused by decreased protein stability. Consistent with this idea, Western analysis showed that Msh4-6A protein levels were lower at all time points during meiosis, averaging a 2.2-fold reduction relative to wild-type Msh4 (Figure 5A,B and Supplemental Figure S5). In contrast, the Msh4-6D protein was hyper-stable, with an average increase of 2.1-fold. However, despite differences in steady-state protein levels, the overall timing of Msh4 appearance and disappearance was quite similar for Msh4, Msh4-6A and Msh4-6D proteins.

Next, we examined whether the lower level of the Msh4-6A protein was due to proteasomemediated degradation. Wild-type and $m s h 4-6 A$ cells were treated with the proteasome inhibitor MG132 two hours after transfer to sporulation medium and Msh4 protein levels were measured at 4, 5 and 6 hrs by Western blot (Figure 5C,D). In the absence of MG132, Msh4-6A protein levels were reduced to $21-44 \%$ of wild-type levels. Treatment with MG132 restored Msh4-6A levels to between $94 \%$ and $125 \%$ of Msh4 levels seen in control wild-type cells. MG132 treatment did not have a significant effect on wild-type Msh4 levels at 4 and 5 hrs, but at 6 hrs levels were 1.8-fold higher than in untreated cells (Figure 5C,D). These data imply that phosphorylation stabilizes Msh4 during meiotic prophase I by protecting it from proteasomal degradation. 
If the primary function of phosphorylation is to stabilize Msh4, then overexpression of phosphorylation-defective Msh4-6A protein should suppress msh4-6A mutant phenotypes. Indeed, overexpression of msh4-6A using the strong, copper-inducible CUP1 promoter restored crossing-over and spore viability to near wild-type levels (Figure $\mathbf{5 E - G}$ ).

\section{The Msh4 N-Terminus Encodes a Portable Degron}

Our analysis points to a model in which Msh4 is an intrinsically unstable protein that is stabilized by phosphorylation of $\mathrm{N}$-terminal residues, thereby activating the crossover function of MutS $\gamma$.

To further test this model, full-length Msh4 and an N-terminally truncated derivative (Msh4$\Delta \mathrm{N} 50$ ) were co-expressed in vegetative (non-meiotic) yeast cells using the CUP1 promoter (Figure 5H-J and Supplemental Figure S5). The steady-state level of full-length Msh4 was 3fold lower than that of Msh4- $\Delta$ N50 and treatment with MG132 showed that this difference was due to proteasomal degradation. By contrast, the N-terminus of Msh5 had no effect on its stability (Supplemental Figure S6). Thus, the N-terminal region of Msh4 possesses degron activity.

Comparison of protein levels in strains co-expressing wild-type Msh4 and Msh4- $\Delta$ N50 versus Msh4-6D and Msh4- $\Delta$ N50 revealed that the phospho-mimetic allele significantly attenuated Nterminal degron activity (Figure $\mathbf{5} \mathbf{K}-\mathbf{M}$ ). Protein half-lives, estimated from cycloheximide chase experiments, were $\sim 14,31$ and 61 minutes respectively for Msh4, Msh4-6D and Msh4- $\Delta$ N50 (Supplemental Figure S5).

To address whether the Msh4 N-terminus has autonomous, portable degron activity, residues 150 of wild-type Msh4 (“Degron”) or a phospho-mimetic derivative (“Degron(6D)”) were fused to GFP and co-expressed in vegetative cells together with wild-type GFP (Figure 5N). The Msh4 
degron destabilized GFP, reducing its half-life from $\sim 59$ to 19 mins (Figure 50,P and

Supplemental Figure S5). By contrast, stability of the phospho-mimetic Degron(6D)-GFP fusion was similar to that of wild-type GFP (half life of $\sim 49$ mins). Together, these data indicate that the N-terminal domain of Msh4 comprises an autonomous degron that is neutralized by phosphorylation.

\section{Msh4 Phosphorylation Occurs In Situ At Sites of Recombination}

The genetic requirements for Msh4 phosphorylation were delineated by performing Western analysis on extracts from mutant strains that are defective for successive steps in meiotic recombination, chromosome pairing and synapsis (Figure 6A). The slow migrating band

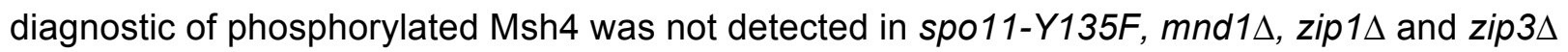
mutants (Figure 6B). The spo11-Y135F allele lacks the catalytic tyrosine required for DSB formation and therefore fails to initiate meiotic recombination (Bergerat et al., 1997). Mnd1 is an essential co-factor for DNA strand-exchange and mnd1 $1 \Delta$ mutant cells are severely defective for DSB repair, homolog pairing and synapsis (Chen et al., 2004; Gerton and DeRisi, 2002;

Tsubouchi and Roeder, 2002). zip1 $1 \Delta$ cells achieve homolog pairing, but formation of crossoverdesignated JMs is defective and synapsis fails because Zip1 is the major component of the SC central region (Borner et al., 2004; Sym et al., 1993). The SUMO E3 ligase, Zip3, accumulates at future crossover sites and facilitates the loading of other ZMM factors, including MutS $\gamma$ (Agarwal and Roeder, 2000; Cheng et al., 2006; Henderson and Keeney, 2004; Shinohara et al., 2008). zip3 $\Delta$ mutants are defective for homolog synapsis, JM formation and crossing over (Agarwal and Roeder, 2000; Borner et al., 2004). Thus, Msh4 phosphorylation is DSBdependent and requires synapsis and the formation of crossover designated JMs.

Analysis of two additional mutants, $n d t 80 \Delta$ and $m / h 3 \Delta$, showed that Msh4 phosphorylation does not require $\mathrm{dHJ}$ resolution or crossing over (Figure 6B). Ndt80 is a transcription factor required 
for meiosis to progress beyond pachytene (Chu and Herskowitz, 1998). ndt80د cells arrest with fully synapsed chromosomes and unresolved JMs (Allers and Lichten, 2001a; Xu et al., 1995). In $n d t 80 \Delta$ cells, phospho-Msh4 accumulated to high levels ( $\geq 60 \%$ of total Msh4) and persisted in arrested cells (Figure 6B). The MutL $\gamma$ complex, comprising MutL homologs Mlh1 and Mlh3, possesses endonuclease activity that is required for the biased resolution of dHJs into crossovers, but not for JM resolution per se (Claeys Bouuaert and Keeney, 2017; Manhart et al., 2017; Ranjha et al., 2014; Rogacheva et al., 2014; Zakharyevich et al., 2012). In mlh3A mutants, chromosomes synapse and normal levels of JMs are formed and resolved, but crossing over is defective (Zakharyevich et al., 2012). Phosphorylation and turnover of Msh4 appeared normal in $m / h 3 \Delta$ cells (Figure 6B).

Overall, our analysis suggests that Msh4 is phosphorylated in situ at sites of recombination, likely when bound to JM intermediates in the context of synapsed chromosomes. Consistent with this inference, chromatin-associated Msh4 was highly enriched for the phosphorylated form relative to soluble Msh4 (Figure 6C,D). Specifically, while total Msh4 protein was roughly equally distributed between chromatin-bound and soluble cell fractions, 95\% of phospho-Msh4 was found in the chromatin-bound fraction (Figure 6C,D).

\section{Msh4 Phosphorylation is Catalyzed by Dbf4-Dependent Kinase, Cdc7}

To identify the kinase(s) responsible for Msh4 phosphorylation, the requirement for candidate kinases was systematically analyzed (Figure 7). The PI3K-like kinases, Mec $1^{\text {ATR }}$ and Tel1 $1^{\text {ATM }}$ are primary sensor kinases of the DNA-damage response and function in meiosis to regulate DSB distribution, inter-homolog template bias and crossing over (Carballo and Cha, 2007; Cooper et al., 2016). Msh4 phosphorylation was reduced more than 2-fold in $P_{C L B 2}-M E C 1$ cells in which the essential MEC1 gene is expressed under the meiotically-repressed CLB2 promoter

(Figure 7A and B)(Lee and Amon, 2003). In contrast, a kinase dead mutant of TEL1 had no 
effect on Msh4 phosphorylation (Figure 7A, (Ma and Greider, 2009)). However, combining $P_{C L B 2}-M E C 1$ with tel1-kd abolished Msh4 phosphorylation and lowered protein levels of Msh4 indicating that TEL1 can partially compensate for the lack of MEC1 with regard to Msh4 phosphorylation.

None of the identified Msh4 phosphorylation sites conform to the S/T-Q Mec1/Tel1 target-site consensus (Figure 1E), making it unlikely to be a direct target. Thus, we explored whether signaling pathways downstream of Mec1/Tel1 are important for Msh4 phosphorylation. An important meiotic target of Mec1/Tel1 is Hop1, a checkpoint adaptor for the meiotic DNA damage response that recruits and activates the serine/threonine effector kinase, Mek1 (Carballo et al., 2008; Niu et al., 2005). Mek1 promotes inter-homolog bias, synapsis and crossing over, and regulates meiotic progression (Callender et al., 2016; Chen et al., 2015; Hollingsworth, 2010; Niu et al., 2007; Prugar et al., 2017; Subramanian et al., 2016; Wu et al., 2010). Although Mek1 consensus-target sites (RXXI) are absent from the Msh4 N-terminus, a mek1 $\Delta$ null mutation abolished Msh4 phosphorylation and reduced steady-state protein levels (Figure 7C). Diminished Msh4 phosphorylation in mek1 $\Delta$ cells could be an indirect effect of the defective inter-homolog interactions caused by this mutation. Therefore, we employed a chemical genetic approach to inhibit Mek1 kinase activity after inter-homolog interactions and synapsis had been established (Figure 7D). Inhibition of the analog-sensitive mek1-as allele (Wan et al., 2004) at $5 \mathrm{hrs}$ after transfer to sporulation medium resulted in the rapid disappearance of phosphorylated Msh4 and a reduction in Msh4 protein levels (Figure 7D and E). To test whether Msh4 is a direct substrate of Mek1, recombinant MutS $\gamma$ was incubated with immuno-purified GST-Mek1-as (Figure 7F). Phosphorylation catalyzed specifically by GSTMek1-as was detected using the ATP $\gamma \mathrm{S}$ analog, $\mathrm{N}^{6}$-Furfuryl-ATPyS, and the semi-synthetic epitope system (Allen et al., 2007; Lo and Hollingsworth, 2011). Under these conditions, only 
auto-phosphorylation of Mek1-as was detected indicating that neither Msh4 or Msh5 is a direct target of Mek1.

Dbf4-dependent kinase (DDK) comprises the kinase Cdc7 and regulatory subunit Dbf4 (Matsumoto and Masai, 2013). DDK functions throughout meiosis and promotes ZMM-mediated crossing over through phosphorylation of Zip1 (Chen et al., 2015). DDK prefers to phosphorylate serines and threonines immediately upstream of a negative charge, which can be conferred either by negatively charged amino acids, such as aspartate or glutamate, or by phosphorylation (Cho et al., 2006; Montagnoli et al., 2006). Msh4 S2 and S7 and S46 are candidates for DDK sites bases on these criteria (Figure 1E). Analogous to mek1-as, inhibition of the analog-sensitive cdc7-as allele (Wan et al., 2006) at $5 \mathrm{hrs}$ (after Cdc7 has activated DSB and SC formation) caused rapid disappearance of phosphorylated Msh4 accompanied by a reduction in total protein level (Figure $\mathbf{7 G}$ and $\mathbf{H}$ ).

In vitro phosphorylation reactions showed that DDK directly phosphorylates the Msh4 Nterminus (Figure 7I). To specifically detect phosphorylation catalyzed by Cdc7, immuno-purified FLAG-Cdc7-as/Dbf4 complex was incubated with 6-Benzyl-ATPYS and phosphorylation was detected via the semi-synthetic epitope system (Allen et al., 2007; Chen et al., 2015; Lo and Hollingsworth, 2011). In the absence of recombinant MutS $\gamma$, only auto-phosphorylation of Cdc7as and Dbf4 was detected. When wild-type MutS $\gamma$ (“WT" in Figure 7I) was added, an additional phosphorylation product was detected that could be either Msh4 or Msh5 based on its molecular weight. The identity of this product was determined in two ways. First, MutS $\gamma$ derivatives containing Msh4-3A ("3A" mutant for S2, S4 and S7) or Msh4-6A ("6A") proteins were used as substrates. Phosphorylation of Msh4-3A was reduced by $68 \%$ relative to wild type, while Msh46A was not modified by DDK, clearly identifying Msh4 as the DDK substrate (Figure 7I). 
Second, when Msh4, Msh4-3A and Msh4-6A proteins were purified from DDK kinase assay mixtures, only the largest phosphorylation product was detected. In addition, all phosphorylation products were sensitive to inhibition of Cdc7-as by the bulky ATP analog, PP1, confirming the specificity of this reaction.

\section{DISCUSSION}

\section{Regulated Proteolysis Is a Key Aspect of Meiotic Crossing Over}

The molecular mechanisms that underpin the differentiation of meiotic crossover and noncrossover pathways have remained elusive. Specifically, it is not known how events leading to $\mathrm{dHJ}$ formation are facilitated at some recombination sites but not at others, and how $\mathrm{dHJs}$ maintain their crossover fate and undergo crossover-biased resolution. Here, regulated proteolysis is revealed as a key determinant of crossing over. This discovery substantiates previous studies implicating the ubiquitin-proteasome system (UPS) in crossover/non-crossover differentiation (Ahuja et al., 2017; Qiao et al., 2014; Rao et al., 2017; Reynolds et al., 2013). Notably, when the UPS is inactivated in mouse spermatocytes, meiotic recombination stalls and ZMM factors (including MutS $\gamma$ ) persist at sites that would normally mature into non-crossovers, suggesting that ZMMs may be targeted for proteolysis at these sites (Rao et al., 2017). At least one ZMM factor, Msh4, can now be designated as a direct target of proteasomal degradation. The atypical mode of Msh4 regulation reveals unanticipated facets of crossover differentiation: intrinsic instability of an essential factor dictates that non-crossover will be the default outcome, and kinase-dependent stabilization activates crossing over.

\section{The Crossover Activity of MutS $\gamma$ Is Activated by Stabilizing Msh4}

Distinct activities of the ZMMs influence different aspects of crossover maturation and couple these events to homolog synapsis. The DNA helicase, Mer3, functions both to regulate the 
extension of nascent D-loops by DNA synthesis and stabilize JMs (Borner et al., 2004; Duroc et al., 2017; Mazina et al., 2004; Nakagawa and Kolodner, 2002); the XPF-ERCC1 related complex, Zip2-Spo16, specifically binds JMs (De Muyt et al., 2018; Guiraldelli et al., 2018; Macaisne et al., 2011); Zip1 acts both locally to promote ZMM function and globally as the major component of SCs (Chen et al., 2015; Sym et al., 1993; Voelkel-Meiman et al., 2015); Zip3 is a SUMO E3 ligase that helps localize other ZMMs to nascent crossover sites and facilitates synapsis (Agarwal and Roeder, 2000; Cheng et al., 2006; Macqueen and Roeder, 2009; Shinohara et al., 2008); and Zip4 is a large TPR repeat protein thought to bridge interactions between Zip2-Spo16, Zip3, MutS $\gamma$ and the chromosome axis protein Red1 (De Muyt et al., 2018). Several activities are ascribed to MutS $\gamma$ : (i) specific binding to JM structures (D-loops and Holliday junctions)(Snowden et al., 2004); (ii) stabilization of nascent JMs following ATPdependent conversion of JM-bound MutS $\gamma$ into sliding clamps that diffuse away from junction points while embracing two DNA duplexes (Snowden et al., 2004); (iii) protection of dHJs from the anti-crossover "dissolution" activity of the STR decatenase complex, Sgs1-Top3-Rmi1 (equivalent to the human BTR complex, BLM-TOPIII $\alpha-$ RMI1/2)(Jessop et al., 2006; Kaur et al., 2015; Oh et al., 2007; Tang et al., 2015)(Tang and Hunter, unpublished); (iv) direct or indirect recruitment and activation of crossover-biased JM resolving factors such as the MutL $\gamma$ endonuclease (Manhart et al., 2017; Nishant et al., 2008; Ranjha et al., 2014; Zakharyevich et al., 2012); (v) formation and/or stabilization of homolog synapsis (Borner et al., 2004; Novak et al., 2001).

Phosphorylation-defective Msh4-6A protein can still localize to chromosomes and retains significant function for synapsis and JM formation. However, the essential crossover function(s) of MutS $\gamma$ is inactive unless Msh4 is stabilized via phosphorylation. We suggest that these essential functions are to protect dHJs from STR/BTR-mediated dissolution and facilitate their 
biased resolution. This proposal is also consonant with our inference that DDK targets MutS $\gamma$ complexes that have bound JMs in the context of synapsed or synapsing chromosomes. Notably, STR/BTR complexes also accumulate at crossover sites (Jagut et al., 2016; Rockmill et al., 2003; Woglar and Villeneuve, 2018); and the symmetric arrangement of dual foci of MutS $\gamma$ and BTR observed in C. elegans suggests a specific model in which MutS $\gamma$ sliding clamps accumulate between the two junctions of a $\mathrm{dHJ}$ to impede dissolution (Woglar and Villeneuve, 2018). We propose that sliding clamps of MutS $\gamma$ must accumulate above a minimum number to facilitate crossing over, be it through $\mathrm{dHJ}$ stabilization, recruitment or activation of resolving enzymes, or maintaining $\mathrm{dHJs}$ in a geometry that is conducive to crossover-biased resolution. Under this model, the requisite threshold of $\mathrm{dHJ}$-bound MutS $\gamma$ clamps requires the stabilization of Msh4 by phosphorylation. We note that the estimated half-life of stabilized Msh4 (30-60 mins) is similar to the estimated lifespan of dHJs (Allers and Lichten, 2001a; Hunter and Kleckner, 2001) suggesting a causal relationship.

If MutS $\gamma$ were the primary limiting factor for crossing over, then the hyper-stable phosphomimetic Msh4-6D protein would be expected to increase crossing over. Although msh4-6D strains showed modest increases in Msh4 foci and IH-dHJs, elevated gene conversion and mildly perturbed interference, crossing over was not increased. Thus, some other factor(s) limits crossover numbers. The most likely interpretation is that crossovers are limited by interference and stabilization of Msh4 occurs at designated crossover sites, downstream of the initial crossover/non-crossover decision. However, the proteolysis mechanism revealed here for Msh4 could be a general mechanism to regulate the availability of essential crossover factors and thereby limit crossovers. 
We further suggest that the intrinsic instability of Msh4 may be enhanced by proximity to proteasomes, which are recruited in high numbers along chromosome axes as they synapse (Ahuja et al., 2017; Rao et al., 2017). This SC-associated population of proteasomes could accelerate the loss of MutS $\gamma$ from synapsed sites where Msh4 is not stabilized by phosphorylation and thereby drive recombination towards a non-crossover outcome.

Whether MutS $\gamma$ is similarly regulated in other organisms remains unclear. An N-terminal region appears to be common to all Msh4 proteins, but sequence conservation is low. However, these regions are typically S/T rich, contain candidate DDK sites and are predicted to undergo disorder-enhanced phosphorylation (Supplemental Figure S1 and data not shown). The intrinsic stability of Msh4 would presumably have to coevolve with the duration of meiotic prophase in different species, which can vary by at least an order of magnitude.

\section{DDK Is A Key Effector of Meiotic Prophase}

In addition to stabilizing Msh4 to activate MutS $\gamma$ for crossing over, DDK facilitates meiotic Sphase (Valentin et al., 2006; Wan et al., 2006); triggers DSBs and couples their formation to the passage of replication forks (Matos et al., 2008; Murakami and Keeney, 2014; Sasanuma et al., 2008; Wan et al., 2008); promotes synapsis and crossing over via phosphorylation of Zip1 on its C-terminus (Chen et al., 2015); enables progression beyond pachytene by removing the Sum1 repression complex from the NDT80 promoter (Lo et al., 2012; Lo et al., 2008); drives the destruction of SCs (Argunhan et al., 2017); is required to recruit monopolin to kinetochores enabling mono-orientation of homologs on the meiosis-I spindle (Lo et al., 2008; Matos et al., 2008); and facilitates the cleavage of cohesin to allow homolog disjunction at the meiosis-I division (Katis et al., 2010). Thus, DDK is a primary effector kinase for all the major events of meiotic prophase. 
Direct targeting of both Zip1 and Msh4 implies that DDK is a general activator of ZMM-mediated crossing over. However, the timing, requirements and modes of regulation are distinct. By contrast to Msh4, Zip1 phosphorylation is an early event that depends on DSB formation but not later steps of recombination and doesn't act by stabilizing the protein (Chen et al., 2015). Moreover, DDK-mediated phosphorylation of Zip1 is inferred to function upstream of the other ZMMs. Consistent with this inference, Msh4 phosphorylation requires both the presence of Zip1 and phosphorylation of its C-terminus (Figure 6 and data not shown). Importantly, the upstream requirement for Mek1 in DDK-mediated Zip1 phosphorylation (Chen et al., 2015) explains why Msh4 phosphorylation is also Mek1 dependent.

It's unclear how DDK achieves successive, dependent phosphorylation of Zip1 and Msh4. An intriguing possibility is that DDK is sequestered by Zip1 until synapsis ensues, a model suggested by recent analysis of substrate ordering in mitotically cycling cells (Seoane and Morgan, 2017). Alternatively, the N-terminal region of Msh4 could be masked until MutS $\gamma$ converts to a sliding clamp on DNA; or crossover-designated recombination complexes could create composite docking sites for DDK. Apparent ordering could involve rapid reversal of DDKcatalyzed phosphorylation until Msh4 becomes protected at designated crossover sites. With this model in mind, Woglar and Villeneuve recently demonstrated that crossover-designated recombination complexes become enveloped in "bubbles" of SC central region proteins that could protect components from both phosphatases and proteasomes (Woglar and Villeneuve, 2018).

\section{Contingent Kinase Cascades Order The Events of Meiotic Prophase}

While DKK appears to be the ultimate effector for many prophase events, other kinases dictate its activity in space and time. CDK primes DDK phosphorylation of Mer2 to trigger DSB 
formation (Henderson et al., 2006; Sasanuma et al., 2008; Wan et al., 2008). DSB-dependent activation of Mec1 $1^{\text {ATR }} /$ Tel $^{\text {ATM }}$ locally activates Mek1 (Carballo et al., 2008), which promotes inter-homolog recombination via its direct targets (Callender et al., 2016; Niu et al., 2009), and indirectly activates synapsis and the ZMM pathway by licensing DDK to phosphorylate Zip1 and subsequently Msh4 (Chen et al., 2015)(this study). CDK, DDK and the meiosis-specific kinase Ime2 collectively target the Sum1 transcriptional repressor to help activate expression of the transcription factor Ndt80 and exit from pachytene. The polo-like kinase Cdc5, whose expression is Ndt80 dependent, then collaborates with CDK and DDK to disassemble SCs (Argunhan et al., 2017; Sourirajan and Lichten, 2008). Cdc5 also collaborates with DDK to localize the monopolin complex to MI kinetochores (Lo et al., 2008; Matos et al., 2008). Finally, casein kinase $\delta / \varepsilon$ works with DDK to activate the cleavage of cohesin by separase and trigger the meiosis-I division (Katis et al., 2010). Understanding the spatial-temporal regulation of DDK with respect to the activation of ZMM-dependent crossing over, and its relationship to crossover control are important goals for the future.

\section{EXPERIMENTAL PROCEDURES}

Extended methods are described in the Supplemental Information.

\section{SUPPLEMENTAL INFORMATION}

Supplemental Information includes 7 figures, 5 tables and extended methods.

\section{ACKNOWLEDGEMENTS}

We thank members of the Hunter Lab for support and discussions. This work was supported by NIH NIGMS grant GM074223 to N.H. and GM050717 to N.M.H. S.T. was supported by an NIH NIEHS-funded training program in Environmental Health Sciences (T32 ES007058). N.H. is an Investigator of the Howard Hughes Medical Institute. 


\section{AUTHOR CONTRIBUTIONS}

W.H. and N.H. conceived the study and designed most experiments. N.M.H., X.C. and W.H. designed in vitro kinase experiments. All authors performed experiments and analyzed the data. W.H. and N.H. wrote the manuscript with inputs and edits from all authors.

\section{DECLARATION OF INTERESTS}

The authors declare no competing interests.

\section{REFERENCES}

Agarwal, S., and Roeder, G.S. (2000). Zip3 provides a link between recombination enzymes and synaptonemal complex proteins. Cell 102, 245-255.

Ahuja, J.S., Sandhu, R., Mainpal, R., Lawson, C., Henley, H., Hunt, P.A., Yanowitz, J.L., and Borner, G.V. (2017). Control of meiotic pairing and recombination by chromosomally tethered 26 S proteasome. Science 355, 408-411.

Allen, J.J., Li, M., Brinkworth, C.S., Paulson, J.L., Wang, D., Hubner, A., Chou, W.H., Davis, R.J., Burlingame, A.L., Messing, R.O., et al. (2007). A semisynthetic epitope for kinase substrates. Nat Methods 4, 511-516.

Allers, T., and Lichten, M. (2001a). Differential timing and control of noncrossover and crossover recombination during meiosis. Cell 106, 47-57.

Allers, T., and Lichten, M. (2001b). Intermediates of yeast meiotic recombination contain heteroduplex DNA. Mol Cell 8, 225-231.

Argunhan, B., Leung, W.K., Afshar, N., Terentyev, Y., Subramanian, V.V., Murayama, Y., Hochwagen, A., Iwasaki, H., Tsubouchi, T., and Tsubouchi, H. (2017). Fundamental cell cycle 
kinases collaborate to ensure timely destruction of the synaptonemal complex during meiosis. EMBO J 36, 2488-2509.

Bergerat, A., de Massy, B., Gadelle, D., Varoutas, P.C., Nicolas, A., and Forterre, P. (1997). An atypical topoisomerase II from Archaea with implications for meiotic recombination. Nature 386, 414-417.

Bhalla, N., Wynne, D.J., Jantsch, V., and Dernburg, A.F. (2008). ZHP-3 acts at crossovers to couple meiotic recombination with synaptonemal complex disassembly and bivalent formation in C. elegans. PLoS Genet 4, e1000235.

Borner, G.V., Kleckner, N., and Hunter, N. (2004). Crossover/noncrossover differentiation, synaptonemal complex formation, and regulatory surveillance at the leptotene/zygotene transition of meiosis. Cell 117, 29-45.

Callender, T.L., Laureau, R., Wan, L., Chen, X., Sandhu, R., Laljee, S., Zhou, S., Suhandynata, R.T., Prugar, E., Gaines, W.A., et al. (2016). Mek1 down regulates Rad51 activity during yeast meiosis by phosphorylation of Hed1. PLoS Genet 12, e1006226.

Carballo, J.A., and Cha, R.S. (2007). Meiotic roles of Mec1, a budding yeast homolog of mammalian ATR/ATM. Chromosome Res 15, 539-550.

Carballo, J.A., Johnson, A.L., Sedgwick, S.G., and Cha, R.S. (2008). Phosphorylation of the axial element protein Hop1 by Mec1/Tel1 ensures meiotic interhomolog recombination. Cell $132,758-770$.

Chelysheva, L., Vezon, D., Chambon, A., Gendrot, G., Pereira, L., Lemhemdi, A., Vrielynck, N., Le Guin, S., Novatchkova, M., and Grelon, M. (2012). The Arabidopsis HEI10 is a new ZMM protein related to Zip3. PLoS Genet 8, e1002799.

Chen, X., Suhandynata, R.T., Sandhu, R., Rockmill, B., Mohibullah, N., Niu, H., Liang, J., Lo, H.C., Miller, D.E., Zhou, H., et al. (2015). Phosphorylation of the synaptonemal complex protein Zip1 regulates the crossover/noncrossover decision during yeast meiosis. PLoS Biol 13, e1002329. 
Chen, Y.K., Leng, C.H., Olivares, H., Lee, M.H., Chang, Y.C., Kung, W.M., Ti, S.C., Lo, Y.H., Wang, A.H., Chang, C.S., et al. (2004). Heterodimeric complexes of Hop2 and Mnd1 function with Dmc1 to promote meiotic homolog juxtaposition and strand assimilation. Proc Natl Acad Sci U S A 101, 10572-10577.

Cheng, C.H., Lo, Y.H., Liang, S.S., Ti, S.C., Lin, F.M., Yeh, C.H., Huang, H.Y., and Wang, T.F. (2006). SUMO modifications control assembly of synaptonemal complex and polycomplex in meiosis of Saccharomyces cerevisiae. Genes Dev 20, 2067-2081.

Cho, W.H., Lee, Y.J., Kong, S.I., Hurwitz, J., and Lee, J.K. (2006). CDC7 kinase phosphorylates serine residues adjacent to acidic amino acids in the minichromosome maintenance 2 protein.

Proc Natl Acad Sci U S A 103, 11521-11526.

Chu, S., and Herskowitz, I. (1998). Gametogenesis in yeast is regulated by a transcriptional cascade dependent on Ndt80. Mol Cell 1, 685-696.

Claeys Bouuaert, C., and Keeney, S. (2017). Distinct DNA-binding surfaces in the ATPase and linker domains of MutLgamma determine its substrate specificities and exert separable functions in meiotic recombination and mismatch repair. PLoS Genet 13, e1006722.

Cooper, T.J., Garcia, V., and Neale, M.J. (2016). Meiotic DSB patterning: A multifaceted process. Cell Cycle 15, 13-21.

De Muyt, A., Jessop, L., Kolar, E., Sourirajan, A., Chen, J., Dayani, Y., and Lichten, M. (2012). BLM helicase ortholog Sgs1 is a central regulator of meiotic recombination intermediate metabolism. Mol Cell 46, 43-53.

De Muyt, A., Pyatnitskaya, A., Andreani, J., Ranjha, L., Ramus, C., Laureau, R., FernandezVega, A., Holoch, D., Girard, E., Govin, J., et al. (2018). A meiotic XPF-ERCC1-like complex recognizes joint molecule recombination intermediates to promote crossover formation. Genes Dev 32, 283-296. 
De Muyt, A., Zhang, L., Piolot, T., Kleckner, N., Espagne, E., and Zickler, D. (2014). E3 ligase Hei10: a multifaceted structure-based signaling molecule with roles within and beyond meiosis. Genes Dev 28, 1111-1123.

de Vries, S.S., Baart, E.B., Dekker, M., Siezen, A., de Rooij, D.G., de Boer, P., and te Riele, H. (1999). Mouse MutS-like protein Msh5 is required for proper chromosome synapsis in male and female meiosis. Genes Dev 13, 523-531.

Dong, H., and Roeder, G.S. (2000). Organization of the yeast Zip1 protein within the central region of the synaptonemal complex. J Cell Biol 148, 417-426.

Drouaud, J., Khademian, H., Giraut, L., Zanni, V., Bellalou, S., Henderson, I.R., Falque, M., and Mezard, C. (2013). Contrasted patterns of crossover and non-crossover at Arabidopsis thaliana meiotic recombination hotspots. PLoS Genet 9, e1003922.

Duroc, Y., Kumar, R., Ranjha, L., Adam, C., Guerois, R., Md Muntaz, K., Marsolier-Kergoat, M.C., Dingli, F., Laureau, R., Loew, D., et al. (2017). Concerted action of the MutLbeta heterodimer and Mer3 helicase regulates the global extent of meiotic gene conversion. Elife 6 . Edelmann, W., Cohen, P.E., Kneitz, B., Winand, N., Lia, M., Heyer, J., Kolodner, R., Pollard, J.W., and Kucherlapati, R. (1999). Mammalian MutS homologue 5 is required for chromosome pairing in meiosis. Nat Genet 21, 123-127.

Fung, J.C., Rockmill, B., Odell, M., and Roeder, G.S. (2004). Imposition of crossover interference through the nonrandom distribution of synapsis initiation complexes. Cell 116, 795802.

Gerton, J.L., and DeRisi, J.L. (2002). Mnd1p: an evolutionarily conserved protein required for meiotic recombination. Proc Natl Acad Sci U S A 99, 6895-6900.

Guillon, H., Baudat, F., Grey, C., Liskay, R.M., and de Massy, B. (2005). Crossover and noncrossover pathways in mouse meiosis. Mol Cell 20, 563-573. 
Guiraldelli, M.F., Felberg, A., Almeida, L.P., Parikh, A., de Castro, R.O., and Pezza, R.J. (2018). SHOC1 is a ERCC4-(HhH)2-like protein, integral to the formation of crossover recombination intermediates during mammalian meiosis. PLoS Genet 14, e1007381.

Henderson, K.A., Kee, K., Maleki, S., Santini, P.A., and Keeney, S. (2006). Cyclin-dependent kinase directly regulates initiation of meiotic recombination. Cell 125, 1321-1332.

Henderson, K.A., and Keeney, S. (2004). Tying synaptonemal complex initiation to the formation and programmed repair of DNA double-strand breaks. Proc Natl Acad Sci U S A 101, 4519-4524.

Herbert, M., Kalleas, D., Cooney, D., Lamb, M., and Lister, L. (2015). Meiosis and maternal aging: insights from aneuploid oocytes and trisomy births. Cold Spring Harb Perspect Biol 7, a017970.

Higgins, J.D., Vignard, J., Mercier, R., Pugh, A.G., Franklin, F.C., and Jones, G.H. (2008).

AtMSH5 partners AtMSH4 in the class I meiotic crossover pathway in Arabidopsis thaliana, but is not required for synapsis. Plant $\mathrm{J} 55,28-39$.

Hillers, K.J., and Villeneuve, A.M. (2003). Chromosome-wide control of meiotic crossing over in C. elegans. Curr Biol 13, 1641-1647.

Hollingsworth, N.M. (2010). Phosphorylation and the creation of interhomolog bias during meiosis in yeast. Cell Cycle 9, 436-437.

Hollingsworth, N.M., Ponte, L., and Halsey, C. (1995). MSH5, a novel MutS homolog, facilitates meiotic reciprocal recombination between homologs in Saccharomyces cerevisiae but not mismatch repair. Genes Dev 9, 1728-1739.

Hunter, N. (2015). Meiotic Recombination: The Essence of Heredity. Cold Spring Harb Perspect Biol 7.

Hunter, N., and Kleckner, N. (2001). The single-end invasion: an asymmetric intermediate at the double-strand break to double-holliday junction transition of meiotic recombination. Cell 106, 5970. 
Jagut, M., Hamminger, P., Woglar, A., Millonigg, S., Paulin, L., Mikl, M., Dello Stritto, M.R., Tang, L., Habacher, C., Tam, A., et al. (2016). Separable Roles for a Caenorhabditis elegans RMI1 Homolog in Promoting and Antagonizing Meiotic Crossovers Ensure Faithful Chromosome Inheritance. PLoS Biol 14, e1002412.

Jantsch, V., Pasierbek, P., Mueller, M.M., Schweizer, D., Jantsch, M., and Loidl, J. (2004). Targeted gene knockout reveals a role in meiotic recombination for ZHP-3, a Zip3-related protein in Caenorhabditis elegans. Mol Cell Biol 24, 7998-8006.

Jeffreys, A.J., and May, C.A. (2004). Intense and highly localized gene conversion activity in human meiotic crossover hot spots. Nat Genet 36, 151-156.

Jessop, L., Rockmill, B., Roeder, G.S., and Lichten, M. (2006). Meiotic chromosome synapsispromoting proteins antagonize the anti-crossover activity of Sgs1. PLoS Genet 2, e155.

Jones, G.H. (1984). The control of chiasma distribution. Symp Soc Exp Biol 38, 293-320.

Katis, V.L., Lipp, J.J., Imre, R., Bogdanova, A., Okaz, E., Habermann, B., Mechtler, K., Nasmyth, K., and Zachariae, W. (2010). Rec8 phosphorylation by casein kinase 1 and Cdc7Dbf4 kinase regulates cohesin cleavage by separase during meiosis. Dev Cell 18, 397-409. Kaur, H., De Muyt, A., and Lichten, M. (2015). Top3-Rmi1 DNA single-strand decatenase is integral to the formation and resolution of meiotic recombination intermediates. Mol Cell 57, 583-594.

Kim, S., Peterson, S.E., Jasin, M., and Keeney, S. (2016). Mechanisms of germ line genome instability. Semin Cell Dev Biol 54, 177-187.

Kneitz, B., Cohen, P.E., Avdievich, E., Zhu, L., Kane, M.F., Hou, H., Jr., Kolodner, R.D., Kucherlapati, R., Pollard, J.W., and Edelmann, W. (2000). MutS homolog 4 localization to meiotic chromosomes is required for chromosome pairing during meiosis in male and female mice. Genes Dev 14, 1085-1097. 
Krishnaprasad, G.N., Anand, M.T., Lin, G., Tekkedil, M.M., Steinmetz, L.M., and Nishant, K.T. (2015). Variation in crossover frequencies perturb crossover assurance without affecting meiotic chromosome segregation in Saccharomyces cerevisiae. Genetics 199, 399-412.

Lam, I., and Keeney, S. (2014). Mechanism and regulation of meiotic recombination initiation. Cold Spring Harb Perspect Biol 7, a016634.

Lao, J.P., Cloud, V., Huang, C.C., Grubb, J., Thacker, D., Lee, C.Y., Dresser, M.E., Hunter, N., and Bishop, D.K. (2013). Meiotic crossover control by concerted action of Rad51-Dmc1 in homolog template bias and robust homeostatic regulation. PLoS Genet 9, e1003978.

Lao, J.P., Oh, S.D., Shinohara, M., Shinohara, A., and Hunter, N. (2008). Rad52 promotes postinvasion steps of meiotic double-strand-break repair. Mol Cell 29, 517-524.

Lee, B.H., and Amon, A. (2003). Role of Polo-like kinase CDC5 in programming meiosis I chromosome segregation. Science 300, 482-486.

Lo, H.C., and Hollingsworth, N.M. (2011). Using the semi-synthetic epitope system to identify direct substrates of the meiosis-specific budding yeast kinase, Mek1. Methods Mol Biol 745, 135-149.

Lo, H.C., Kunz, R.C., Chen, X., Marullo, A., Gygi, S.P., and Hollingsworth, N.M. (2012). Cdc7Dbf4 is a gene-specific regulator of meiotic transcription in yeast. Mol Cell Biol 32, 541-557. Lo, H.C., Wan, L., Rosebrock, A., Futcher, B., and Hollingsworth, N.M. (2008). Cdc7-Dbf4 regulates NDT80 transcription as well as reductional segregation during budding yeast meiosis. Mol Biol Cell 19, 4956-4967.

Lynn, A., Soucek, R., and Borner, G.V. (2007). ZMM proteins during meiosis: Crossover artists at work. Chromosome Res 15, 591-605.

Ma, Y., and Greider, C.W. (2009). Kinase-independent functions of TEL1 in telomere maintenance. Mol Cell Biol 29, 5193-5202.

Macaisne, N., Vignard, J., and Mercier, R. (2011). SHOC1 and PTD form an XPF-ERCC1-like complex that is required for formation of class I crossovers. J Cell Sci 124, 2687-2691. 
Macqueen, A.J., and Roeder, G.S. (2009). Fpr3 and Zip3 ensure that initiation of meiotic recombination precedes chromosome synapsis in budding yeast. Curr Biol 19, 1519-1526. Malkova, A., Swanson, J., German, M., McCusker, J.H., Housworth, E.A., Stahl, F.W., and Haber, J.E. (2004). Gene conversion and crossing over along the 405-kb left arm of Saccharomyces cerevisiae chromosome VII. Genetics 168, 49-63.

Manhart, C.M., and Alani, E. (2016). Roles for mismatch repair family proteins in promoting meiotic crossing over. DNA Repair (Amst) 38, 84-93.

Manhart, C.M., Ni, X., White, M.A., Ortega, J., Surtees, J.A., and Alani, E. (2017). The mismatch repair and meiotic recombination endonuclease Mlh1-Mlh3 is activated by polymer formation and can cleave DNA substrates in trans. PLoS Biol 15, e2001164.

Marsolier-Kergoat, M.C., Khan, M.M., Schott, J., Zhu, X., and Llorente, B. (2018). Mechanistic view and genetic control of DNA recombination during meiosis. Mol Cell 70, 9-20 e26.

Martini, E., Borde, V., Legendre, M., Audic, S., Regnault, B., Soubigou, G., Dujon, B., and Llorente, B. (2011). Genome-wide analysis of heteroduplex DNA in mismatch repair-deficient yeast cells reveals novel properties of meiotic recombination pathways. PLoS Genet 7 , e1002305.

Matos, J., Lipp, J.J., Bogdanova, A., Guillot, S., Okaz, E., Junqueira, M., Shevchenko, A., and Zachariae, W. (2008). Dbf4-dependent CDC7 kinase links DNA replication to the segregation of homologous chromosomes in meiosis I. Cell 135, 662-678.

Matsumoto, S., and Masai, H. (2013). Regulation of chromosome dynamics by Hsk1/Cdc7 kinase. Biochem Soc Trans 41, 1712-1719.

Mazina, O.M., Mazin, A.V., Nakagawa, T., Kolodner, R.D., and Kowalczykowski, S.C. (2004). Saccharomyces cerevisiae Mer3 helicase stimulates 3'-5' heteroduplex extension by Rad51; implications for crossover control in meiotic recombination. Cell 117, 47-56.

McMahill, M.S., Sham, C.W., and Bishop, D.K. (2007). Synthesis-dependent strand annealing in meiosis. PLoS Biol 5, e299. 
Montagnoli, A., Valsasina, B., Brotherton, D., Troiani, S., Rainoldi, S., Tenca, P., Molinari, A., and Santocanale, C. (2006). Identification of Mcm2 phosphorylation sites by S-phase-regulating kinases. J Biol Chem 281, 10281-10290.

Murakami, H., and Keeney, S. (2014). Temporospatial coordination of meiotic DNA replication and recombination via DDK recruitment to replisomes. Cell 158, 861-873.

Nakagawa, T., and Kolodner, R.D. (2002). Saccharomyces cerevisiae Mer3 is a DNA helicase involved in meiotic crossing over. Mol Cell Biol 22, 3281-3291.

Nishant, K.T., Chen, C., Shinohara, M., Shinohara, A., and Alani, E. (2010). Genetic analysis of baker's yeast Msh4-Msh5 reveals a threshold crossover level for meiotic viability. PLoS Genet 6.

Nishant, K.T., Plys, A.J., and Alani, E. (2008). A mutation in the putative MLH3 endonuclease domain confers a defect in both mismatch repair and meiosis in Saccharomyces cerevisiae. Genetics 179, 747-755.

Niu, H., Li, X., Job, E., Park, C., Moazed, D., Gygi, S.P., and Hollingsworth, N.M. (2007). Mek1 kinase is regulated to suppress double-strand break repair between sister chromatids during budding yeast meiosis. Mol Cell Biol 27, 5456-5467.

Niu, H., Wan, L., Baumgartner, B., Schaefer, D., Loidl, J., and Hollingsworth, N.M. (2005). Partner choice during meiosis is regulated by Hop1-promoted dimerization of Mek1. Mol Biol Cell 16, 5804-5818.

Niu, H., Wan, L., Busygina, V., Kwon, Y., Allen, J.A., Li, X., Kunz, R.C., Kubota, K., Wang, B., Sung, P., et al. (2009). Regulation of meiotic recombination via Mek1-mediated Rad54 phosphorylation. Mol Cell 36, 393-404.

Novak, J.E., Ross-Macdonald, P.B., and Roeder, G.S. (2001). The budding yeast Msh4 protein functions in chromosome synapsis and the regulation of crossover distribution. Genetics 158, 1013-1025. 
Oh, S.D., Lao, J.P., Hwang, P.Y., Taylor, A.F., Smith, G.R., and Hunter, N. (2007). BLM ortholog, Sgs1, prevents aberrant crossing-over by suppressing formation of multichromatid joint molecules. Cell 130, 259-272.

Papazian, H.P. (1952). The analysis of tetrad data. Genetics 37, 175-188.

Pochart, P., Woltering, D., and Hollingsworth, N.M. (1997). Conserved properties between functionally distinct MutS homologs in yeast. J Biol Chem 272, 30345-30349.

Prugar, E., Burnett, C., Chen, X., and Hollingsworth, N.M. (2017). Coordination of double strand break repair and meiotic progression in yeast by a Mek1-Ndt80 negative feedback loop.

Genetics 206, 497-512.

Qiao, H., Prasada Rao, H.B., Yang, Y., Fong, J.H., Cloutier, J.M., Deacon, D.C., Nagel, K.E., Swartz, R.K., Strong, E., Holloway, J.K., et al. (2014). Antagonistic roles of ubiquitin ligase HEI10 and SUMO ligase RNF212 regulate meiotic recombination. Nat Genet 46, 194-199. Rakshambikai, R., Srinivasan, N., and Nishant, K.T. (2013). Structural insights into Saccharomyces cerevisiae Msh4-Msh5 complex function using homology modeling. PLoS One 8, e78753.

Ranjha, L., Anand, R., and Cejka, P. (2014). The Saccharomyces cerevisiae Mlh1-Mlh3 heterodimer is an endonuclease that preferentially binds to Holliday junctions. J Biol Chem 289, 5674-5686.

Rao, H.B., Qiao, H., Bhatt, S.K., Bailey, L.R., Tran, H.D., Bourne, S.L., Qiu, W., Deshpande, A., Sharma, A.N., Beebout, C.J., et al. (2017). A SUMO-ubiquitin relay recruits proteasomes to chromosome axes to regulate meiotic recombination. Science 355, 403-407.

Reynolds, A., Qiao, H., Yang, Y., Chen, J.K., Jackson, N., Biswas, K., Holloway, J.K., Baudat, F., de Massy, B., Wang, J., et al. (2013). RNF212 is a dosage-sensitive regulator of crossingover during mammalian meiosis. Nat Genet 45, 269-278.

Rockmill, B., Fung, J.C., Branda, S.S., and Roeder, G.S. (2003). The Sgs1 helicase regulates chromosome synapsis and meiotic crossing over. Curr Biol 13, 1954-1962. 
Rockmill, B., Lefrancois, P., Voelkel-Meiman, K., Oke, A., Roeder, G.S., and Fung, J.C. (2013). High throughput sequencing reveals alterations in the recombination signatures with diminishing Spo11 activity. PLoS Genet 9, e1003932.

Rogacheva, M.V., Manhart, C.M., Chen, C., Guarne, A., Surtees, J., and Alani, E. (2014). Mlh1Mlh3, a meiotic crossover and DNA mismatch repair factor, is a Msh2-Msh3-stimulated endonuclease. J Biol Chem 289, 5664-5673.

Sasanuma, H., Hirota, K., Fukuda, T., Kakusho, N., Kugou, K., Kawasaki, Y., Shibata, T., Masai, H., and Ohta, K. (2008). Cdc7-dependent phosphorylation of Mer2 facilitates initiation of yeast meiotic recombination. Genes Dev 22, 398-410.

Seoane, A.I., and Morgan, D.O. (2017). Firing of replication origins frees Dbf4-Cdc7 to target Eco1 for destruction. Curr Biol 27, 2849-2855 e2842.

Shinohara, M., Oh, S.D., Hunter, N., and Shinohara, A. (2008). Crossover assurance and crossover interference are distinctly regulated by the ZMM proteins during yeast meiosis. Nat Genet 40, 299-309.

Snowden, T., Acharya, S., Butz, C., Berardini, M., and Fishel, R. (2004). hMSH4-hMSH5 recognizes Holliday Junctions and forms a meiosis-specific sliding clamp that embraces homologous chromosomes. Mol Cell 15, 437-451.

Sourirajan, A., and Lichten, M. (2008). Polo-like kinase Cdc5 drives exit from pachytene during budding yeast meiosis. Genes Dev 22, 2627-2632.

Stahl, F.W., Foss, H.M., Young, L.S., Borts, R.H., Abdullah, M.F., and Copenhaver, G.P. (2004). Does crossover interference count in Saccharomyces cerevisiae? Genetics 168, 35-48.

Subramanian, V.V., MacQueen, A.J., Vader, G., Shinohara, M., Sanchez, A., Borde, V., Shinohara, A., and Hochwagen, A. (2016). Chromosome synapsis alleviates Mek1-dependent suppression of meiotic DNA repair. PLoS Biol 14, e1002369.

Sym, M., Engebrecht, J.A., and Roeder, G.S. (1993). ZIP1 is a synaptonemal complex protein required for meiotic chromosome synapsis. Cell 72, 365-378. 
Sym, M., and Roeder, G.S. (1995). Zip1-induced changes in synaptonemal complex structure and polycomplex assembly. J Cell Biol 128, 455-466.

Tang, S., Wu, M.K., Zhang, R., and Hunter, N. (2015). Pervasive and essential roles of the Top3-Rmi1 decatenase orchestrate recombination and facilitate chromosome segregation in meiosis. Mol Cell 57, 607-621.

Thacker, D., Mohibullah, N., Zhu, X., and Keeney, S. (2014). Homologue engagement controls meiotic DNA break number and distribution. Nature 510, 241-246.

Tsubouchi, H., and Roeder, G.S. (2002). The Mnd1 protein forms a complex with hop2 to promote homologous chromosome pairing and meiotic double-strand break repair. Mol Cell Biol 22, 3078-3088.

Valentin, G., Schwob, E., and Della Seta, F. (2006). Dual role of the Cdc7-regulatory protein Dbf4 during yeast meiosis. J Biol Chem 281, 2828-2834.

Voelkel-Meiman, K., Johnston, C., Thappeta, Y., Subramanian, V.V., Hochwagen, A., and MacQueen, A.J. (2015). Separable Crossover-Promoting and Crossover-Constraining Aspects of Zip1 Activity during Budding Yeast Meiosis. PLoS Genet 11, e1005335.

Wan, L., de los Santos, T., Zhang, C., Shokat, K., and Hollingsworth, N.M. (2004). Mek1 kinase activity functions downstream of RED1 in the regulation of meiotic double strand break repair in budding yeast. Mol Biol Cell 15, 11-23.

Wan, L., Niu, H., Futcher, B., Zhang, C., Shokat, K.M., Boulton, S.J., and Hollingsworth, N.M. (2008). Cdc28-Clb5 (CDK-S) and Cdc7-Dbf4 (DDK) collaborate to initiate meiotic recombination in yeast. Genes Dev 22, 386-397.

Wan, L., Zhang, C., Shokat, K.M., and Hollingsworth, N.M. (2006). Chemical inactivation of cdc7 kinase in budding yeast results in a reversible arrest that allows efficient cell synchronization prior to meiotic recombination. Genetics $174,1767-1774$.

Wang, K., Wang, M., Tang, D., Shen, Y., Miao, C., Hu, Q., Lu, T., and Cheng, Z. (2012). The role of rice HEI10 in the formation of meiotic crossovers. PLoS Genet 8, e1002809. 
Wang, S., Hassold, T., Hunt, P., White, M.A., Zickler, D., Kleckner, N., and Zhang, L. (2017). Inefficient crossover maturation underlies elevated aneuploidy in human female meiosis. Cell 168, 977-989 e917.

Wang, S., Zickler, D., Kleckner, N., and Zhang, L. (2015). Meiotic crossover patterns: obligatory crossover, interference and homeostasis in a single process. Cell Cycle 14, 305-314.

Watanabe, Y. (2012). Geometry and force behind kinetochore orientation: lessons from meiosis. Nat Rev Mol Cell Biol 13, 370-382.

Wijnker, E., Velikkakam James, G., Ding, J., Becker, F., Klasen, J.R., Rawat, V., Rowan, B.A., de Jong, D.F., de Snoo, C.B., Zapata, L., et al. (2013). The genomic landscape of meiotic crossovers and gene conversions in Arabidopsis thaliana. Elife 2, e01426.

Woglar, A., and Villeneuve, A.M. (2018). Dynamic architecture of DNA repair complexes and the synaptonemal complex at sites of meiotic recombination. Cell 173, 1678-1691 e1616.

Wu, H.Y., Ho, H.C., and Burgess, S.M. (2010). Mek1 kinase governs outcomes of meiotic recombination and the checkpoint response. Curr Biol 20, 1707-1716.

Xu, L., Ajimura, M., Padmore, R., Klein, C., and Kleckner, N. (1995). NDT80, a meiosis-specific gene required for exit from pachytene in Saccharomyces cerevisiae. Mol Cell Biol 15, 65726581.

Yang, W., Junop, M.S., Ban, C., Obmolova, G., and Hsieh, P. (2000). DNA mismatch repair: from structure to mechanism. Cold Spring Harb Symp Quant Biol 65, 225-232.

Yokoo, R., Zawadzki, K.A., Nabeshima, K., Drake, M., Arur, S., and Villeneuve, A.M. (2012). COSA-1 reveals robust homeostasis and separable licensing and reinforcement steps governing meiotic crossovers. Cell 149, 75-87.

Zakharyevich, K., Ma, Y., Tang, S., Hwang, P.Y., Boiteux, S., and Hunter, N. (2010). Temporally and biochemically distinct activities of Exo1 during meiosis: double-strand break resection and resolution of double Holliday junctions. Mol Cell 40, 1001-1015. 
Zakharyevich, K., Tang, S., Ma, Y., and Hunter, N. (2012). Delineation of joint molecule resolution pathways in meiosis identifies a crossover-specific resolvase. Cell 149, 334-347.

Zhang, L., Kohler, S., Rillo-Bohn, R., and Dernburg, A.F. (2018). A compartmentalized signaling network mediates crossover control in meiosis. Elife 7, e30789 doi: 30710.37554/eLife.30789.

Zhang, L., Tang, D., Luo, Q., Chen, X., Wang, H., Li, Y., and Cheng, Z. (2014). Crossover formation during rice meiosis relies on interaction of OsMSH4 and OsMSH5. Genetics 198, 1447-1456.

Zickler, D., and Kleckner, N. (2015). Recombination, pairing, and synapsis of homologs during meiosis. Cold Spring Harb Perspect Biol 7, pii: a016626. doi:

016610.011101/cshperspect.a016626. 
A

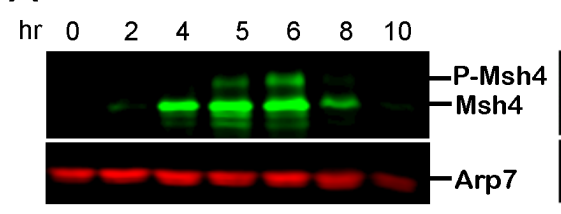

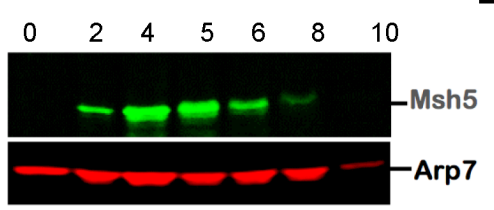

B

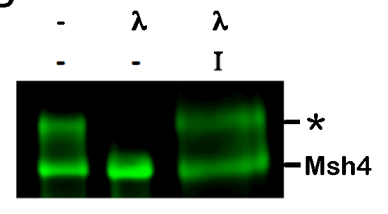

C — total Msh4 - phospho-Msh4 -MI \pm MII
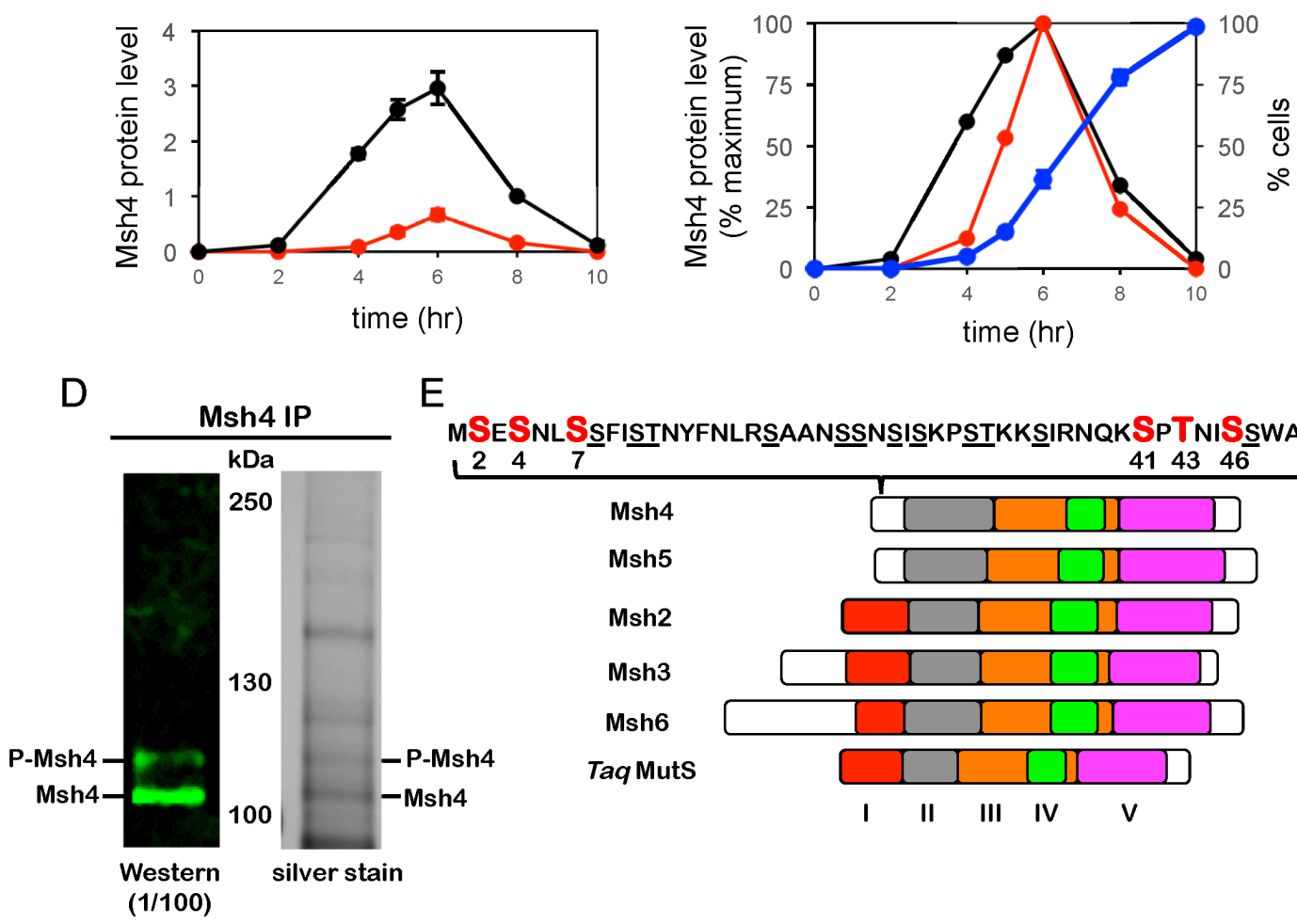

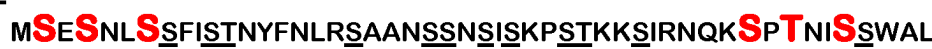
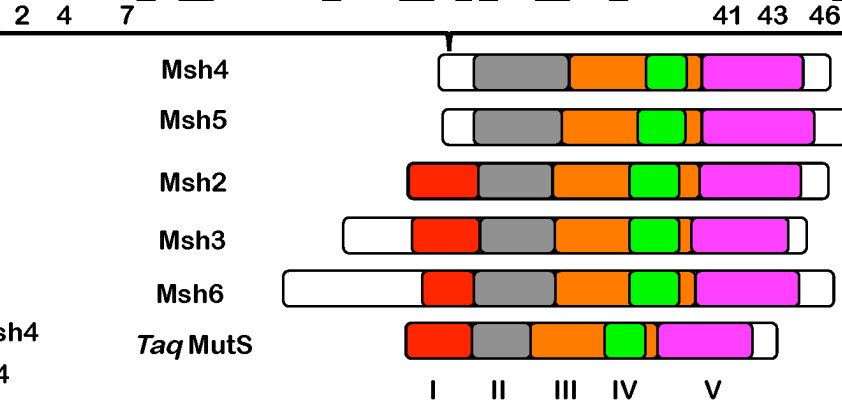

Figure 1. The N-terminal region of Msh4 is phosphorylated.

(A) Western analysis of Msh4 (left) and Msh5 (right) throughout meiosis. Arp7 is used throughout as a loading control (Sourirajan and Lichten, 2008).

(B) Lambda phosphatase treatment of immuno-precipitated Msh4. $\lambda$, phosphatase; I, phosphatase inhibitor.

(C) Relative (left) and normalized (right) levels Msh4 phosphorylation. MI $\pm \mathrm{MII}$ is the percentage of cells that have completed one or both meiotic divisions. Error bars show mean \pm S.E. from four independent time courses. 
bioRxiv preprint doi: https://doi.org/10.1101/386458; this version posted August 27, 2018. The copyright holder for this preprint (which was not certified by peer review) is the author/funder, who has granted bioRxiv a license to display the preprint in perpetuity. It is made available under aCC-BY-NC-ND 4.0 International license.

(D) Western blotting and silver-stained gel images of immunoprecipitated material used for LCMS/MS analysis. The positions of the Msh4 bands that were excised and processed for LCMS/MS are indicated.

(E) Positions of phosphorylation sites (red) mapped by LC-MS/MS. Underlined residues

highlight the high S/T content of the Msh4 N-terminal region. Diagrams show protein domains of eukaryotic nuclear MutS homologs relative to Thermus aquaticus MutS (adapted from (Nishant et al., 2010). Also see Figure S1. 
A

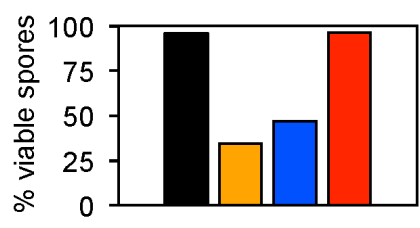

C

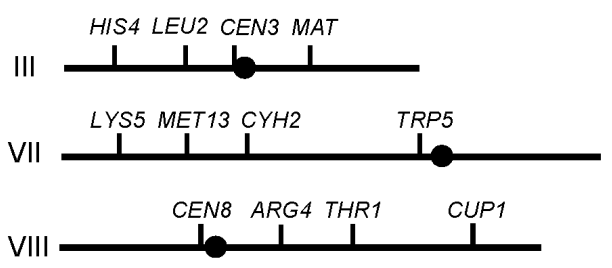

E

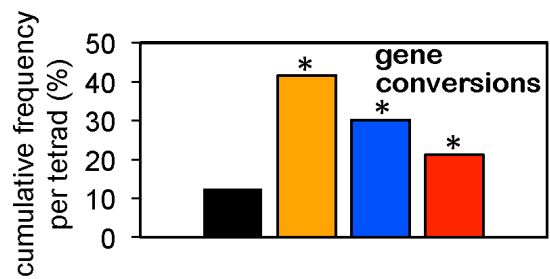

MSH4

$m s h 4 \Delta$ $m s h 46 A$

$m s h 46 D$

D

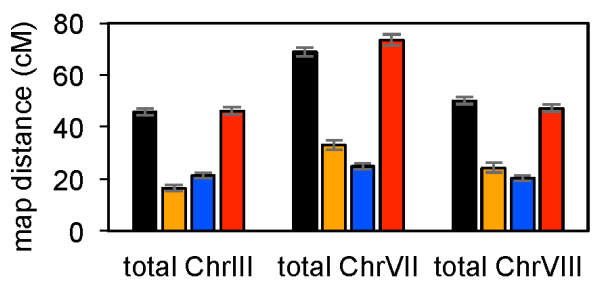

G

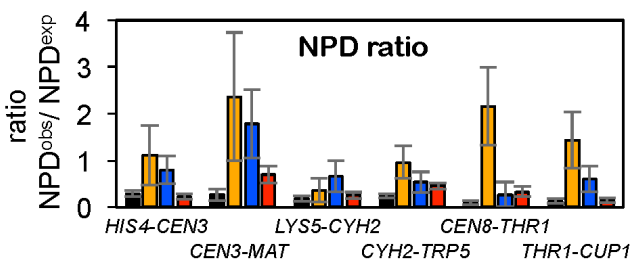

F ${ }_{M S H 4}$

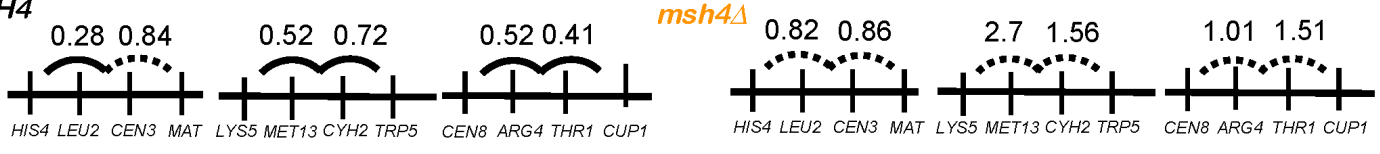

$m s h 4-6 A$
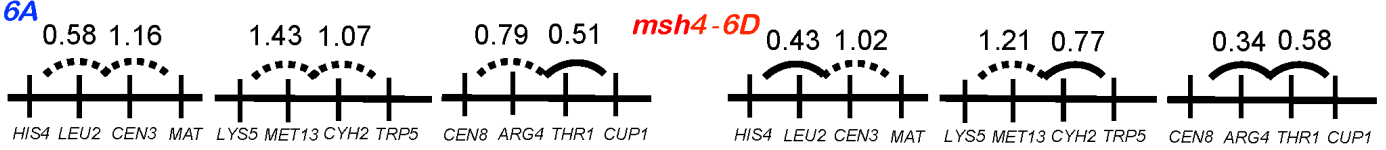

$\mathrm{H}$
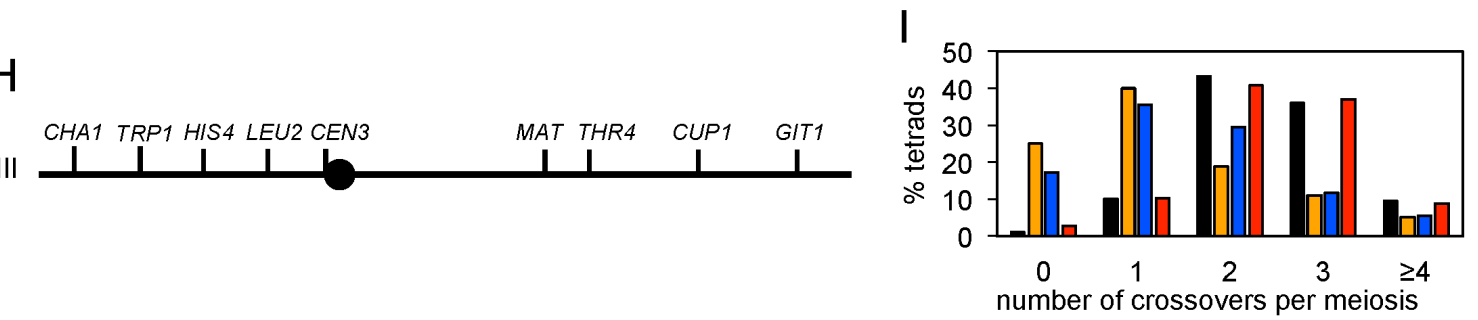

Figure 2. Phosphorylation is essential for the crossover function of Msh4.

(A) Spore viabilities of indicated strains (see Table S3).

(B) Distributions of tetrads with 4, 3, 2, 1 and 0 viable spores. 
(C) Marker configurations in strains used to analyze recombination. CEN3 is marked with the ADE2 gene and CEN8 is marked with URA3 (Oh et al., 2007).

(D) Cumulative map distances ( \pm S.E.) for intervals on chromosomes III, VII and VIII.

(E) Cumulative frequencies of tetrads with gene conversions (non 2:2 segregations) for markers shown in (E). Asterisks indicate $P<0.01$ relative to wild type ( $z$-test).

(F) Interference analysis for adjacent intervals (Lao et al., 2013; Malkova et al., 2004). Solid arcs between intervals indicate significant positive interference; failure to detect significant positive interference is indicated by dashed arcs (see Table S4).

(G) Interference analysis within individual intervals expressed as NPD ratios (https://elizabethhousworth.com/StahlLabOnlineTools/). Error bars show S.E. Asterisks indicate significant positive interference (see Table S6).

(H) Marker configuration in strains used to analyze crossover assurance for chromosome III. CEN3 is heterozgously marked with the LYS2 and URA3 (see Table S1).

(I) Distributions of crossover classes for chromosome III.

See also Figures $\mathbf{S 2}$ and $\mathbf{S 3 .}$ 

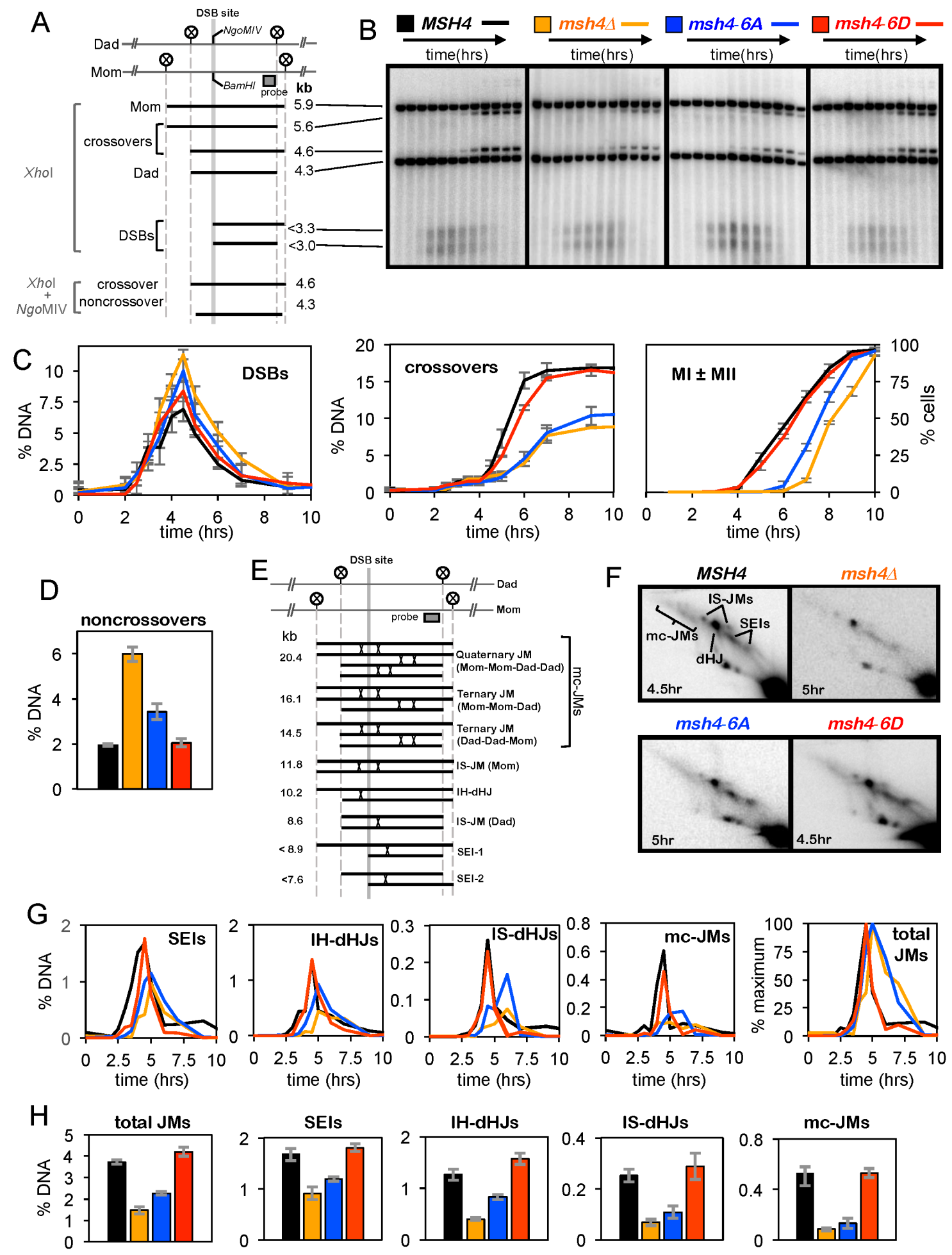

Figure 3. Physical analysis of the DNA events of meiotic recombination.

(A) Map of HIS4:LEU2 locus highlighting the DSB site, Xhol restriction sites (circled Xs) and the position of the probe used in Southern blotting. Sizes of diagnostic fragments are shown below. 
(B) Representative 1D gel Southern blot images for analysis of DSBs and crossovers. Time points are $0,2,2.5,3,3.5,4,4.5,5,6,7,9$ and 11 hours.

(C) Quantification of DSBs, crossovers and meiotic divisions. \%DNA is percentage of total hybridizing DNA signal. MI \pm MII is the percentage of cells that have completed one or both meiotic divisions.

(D) Non-crossover levels at $11 \mathrm{hrs}$.

(E) JM structures detected at the HIS4:LEU2 locus. Positions of the DSB site, diagnostic Xhol sites (circled Xs) and the Southern probe are shown.

(F) Representative 2D gel Southern blot images for time points where JM levels peak. Positions of the various JM signals are indicated in the first panel.

(G) Quantification of JM species over time. (H) Quantification of JM species at their peak levels from three independent time courses. IH-dHJs, inter-homolog dHJs; IS-JMs, intersister JMs (most likely dHJs); SEls, single-end invasions; mc-JMs, multi-chromatid JMs) and total JMs are indicated. Averages \pm S.E. were calculated from three independent experiments. 

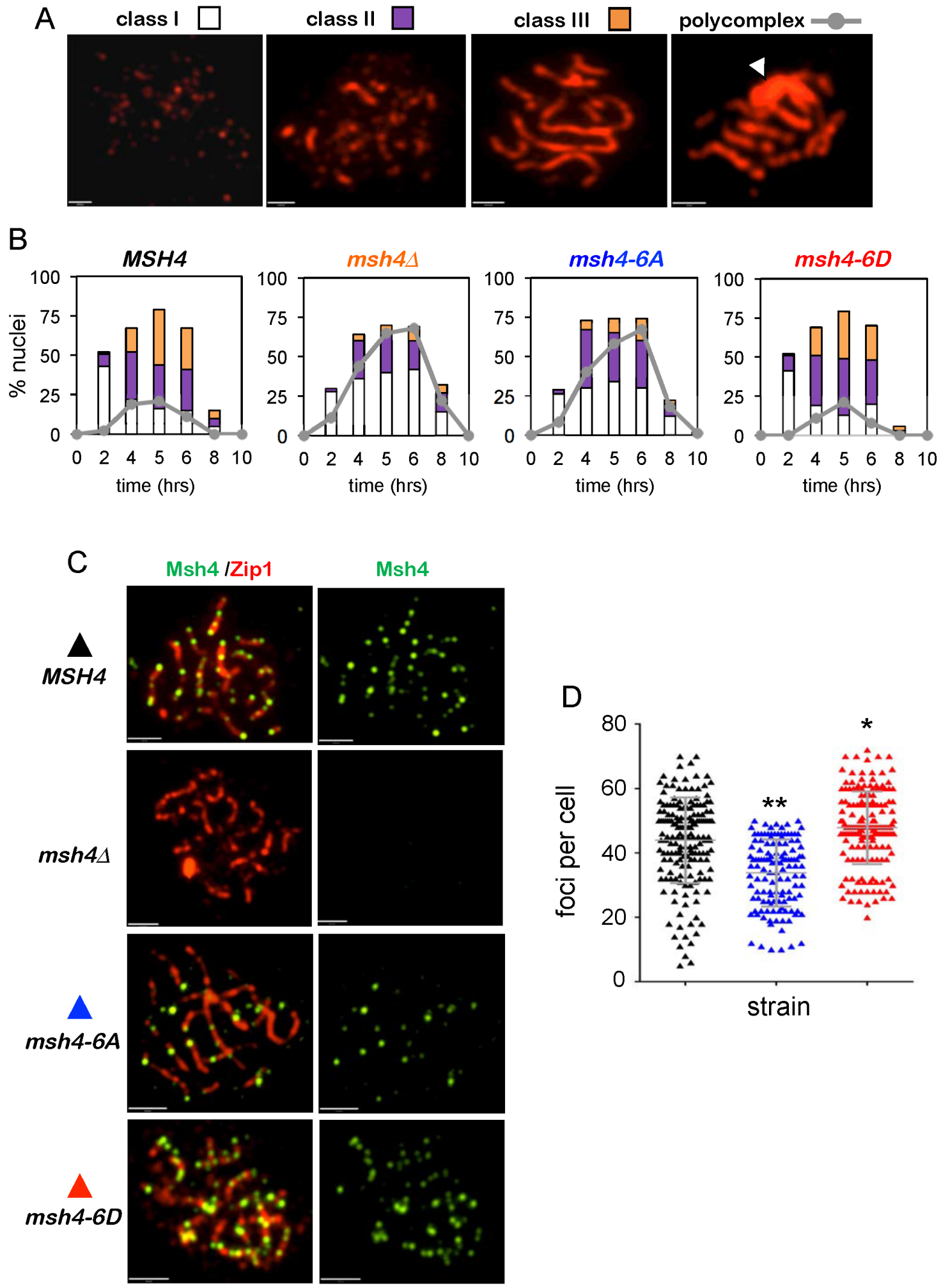

Figure 4. Chromosome synapsis and localization of Msh4 are facilitated by Msh4 phosphorylation. 
bioRxiv preprint doi: https://doi.org/10.1101/386458; this version posted August 27, 2018. The copyright holder for this preprint (which was not certified by peer review) is the author/funder, who has granted bioRxiv a license to display the preprint in perpetuity. It is made available under aCC-BY-NC-ND 4.0 International license.

(A) Chromosome spreads showing representative examples of the three different Zip1 immunostaining classes and a Zip1 polycomplex.

(B) Quantification of Zip1 staining classes and polycomplexes. $\geq 100$ nuclei were scored for each time point.

(C) Representative images of spread meiotic nuclei immuno-stained for Msh4 (green) and Zip1 (red).

(D) Quantification of Msh4 immuno-staining foci in class II and class III nuclei. $\geq 100$ nuclei were scored for each strain. ${ }^{*} P<0.05 ;{ }^{*} P<0.01$ two tailed Mann Whitney test. Scale bars $=30 \mu \mathrm{m}$.

Also see Figure S4. 

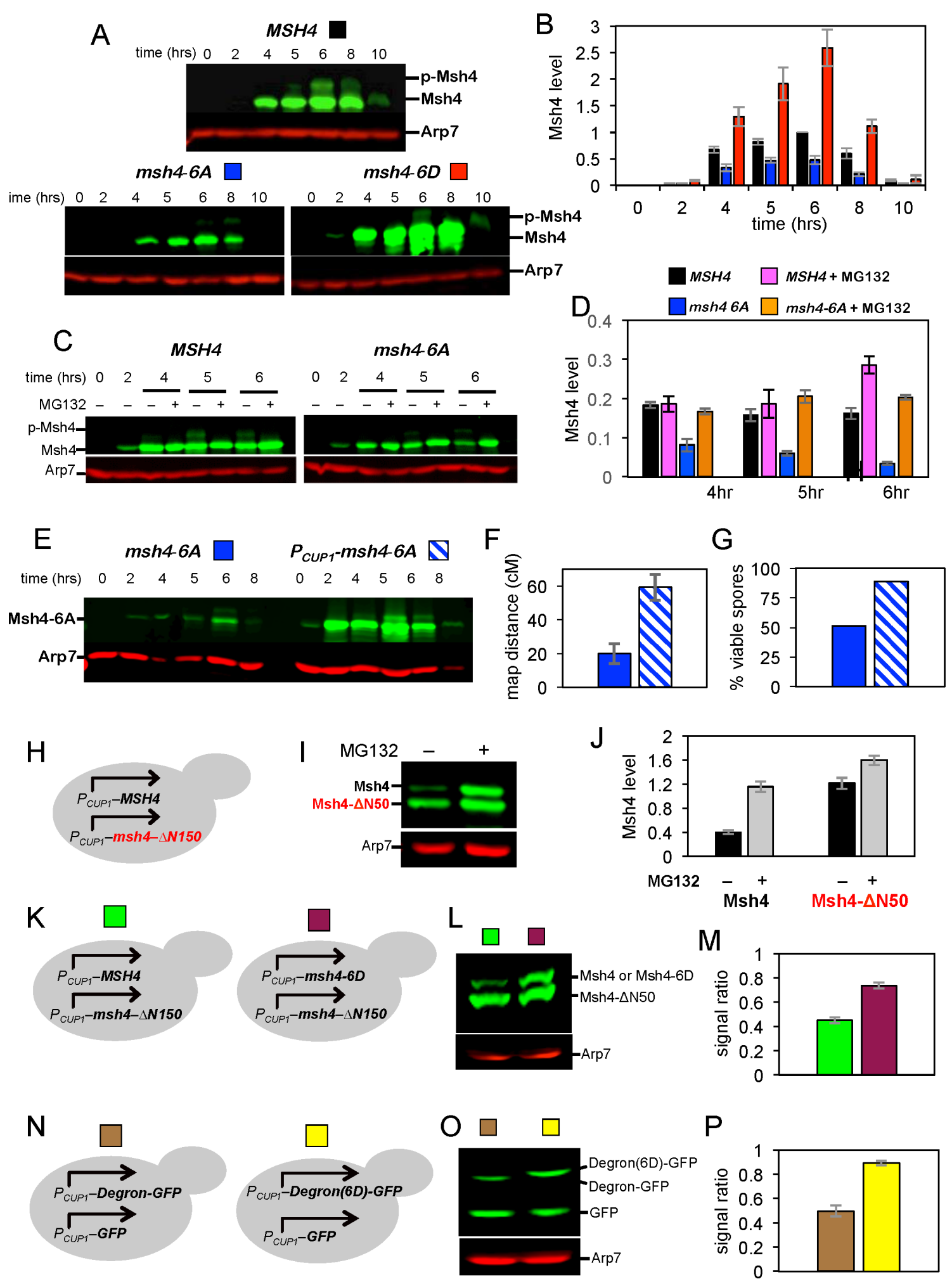

Figure 5. Degron activity of the Msh4 $\mathrm{N}$-terminal region is attenuated by phosphorylation

(A) Western analysis of Msh4 during meiosis in wild-type, $m s h-6 A$ and $m s h 4-6 D$ strains. 
(B) Quantification of Msh4 protein relative to the Arp7 loading control. Averages \pm S.E. were calculated from three independent experiments.

(C) Western analysis of Msh4 with and without addition of the proteasome inhibitor, MG132, at 2 hrs.

(D) Quantification of Msh4 protein with and without MG132 treatment. Averages \pm S.E. calculated from three independent experiments.

(E) Western analysis of Msh4-6A protein during meiosis in the msh4-6A strain and following copper-induced overexpression in a $P_{C U P 1}-m s h 4-6 A$ strain.

(F) Spore viability of $m s h 4-6 A$ and $P_{C U P 1}-m s h 4-6 A$ strains.

(G) Map distances ( \pm S.E.) for intervals flanking the HIS4::LEU2 recombination hotspot (see Figure S3) in tetrads from $m s h 4-6 A$ and $P_{C U P 1}-m s h 4-6 A$ strains.

(H) Experimental system for copper-inducible expression of Msh4 and msh4- $\Delta$ N50 proteins in vegetative cells.

(I) Western analysis of the stains shown in panel E following copper induction, with and without MG132 treatment.

(J) Quantification of the experiments represented in panels D and E. Averages \pm S.E. were calculated from four independent experiments.

(K) Experimental systems to compare co-expression of Msh4 and Msh4- $\Delta$ N50 (left), with coexpression of Msh4-6D and Msh4- $\Delta$ N50 proteins (right).

(L) Western analysis of the stains shown in panel $\mathrm{H}$ following copper induction, with and without MG132 treatment.

(M) Quantification of experiments represented in panels $\mathrm{H}$ and I. Average ratios \pm S.E. were calculated from four independent experiments.

(N) Experimental systems for co-expression of GFP and the Msh4 N-terminal region ("degron") fused to GFP (left); or co-expresson of GFP and a phospho-mimetic derivative of the Msh4 Nterminal region ("degron 6D) fused to GFP. 
bioRxiv preprint doi: https://doi.org/10.1101/386458; this version posted August 27, 2018. The copyright holder for this preprint (which was not certified by peer review) is the author/funder, who has granted bioRxiv a license to display the preprint in perpetuity. It is made available under aCC-BY-NC-ND 4.0 International license.

(O) Western analysis of the stains shown in panel $\mathrm{K}$ following copper induction.

(P) Quantification of experiments represented in panels $\mathrm{K}$ and L. Average ratios \pm S.E. were calculated from four independent experiments. Also see Figure S5. 

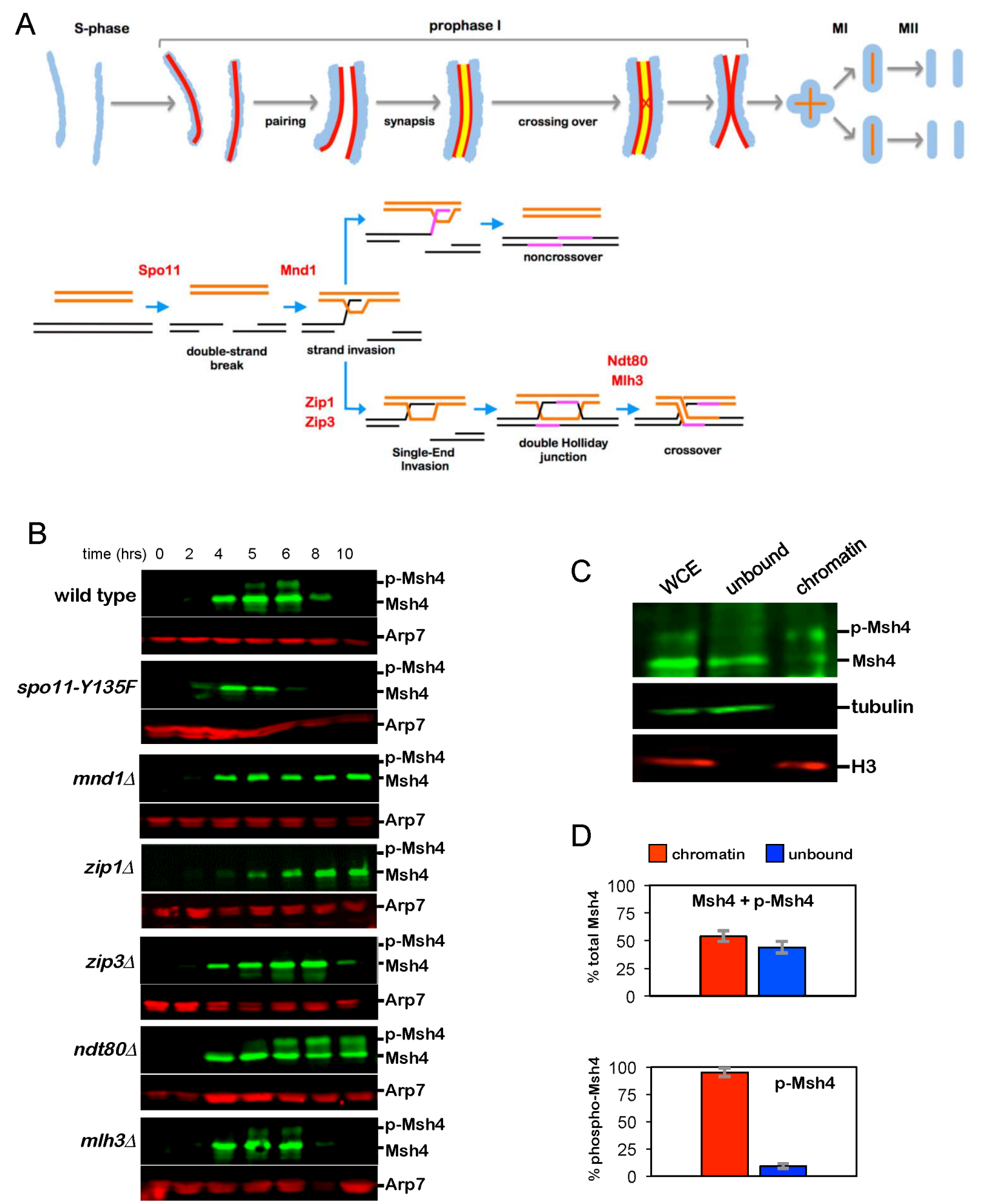
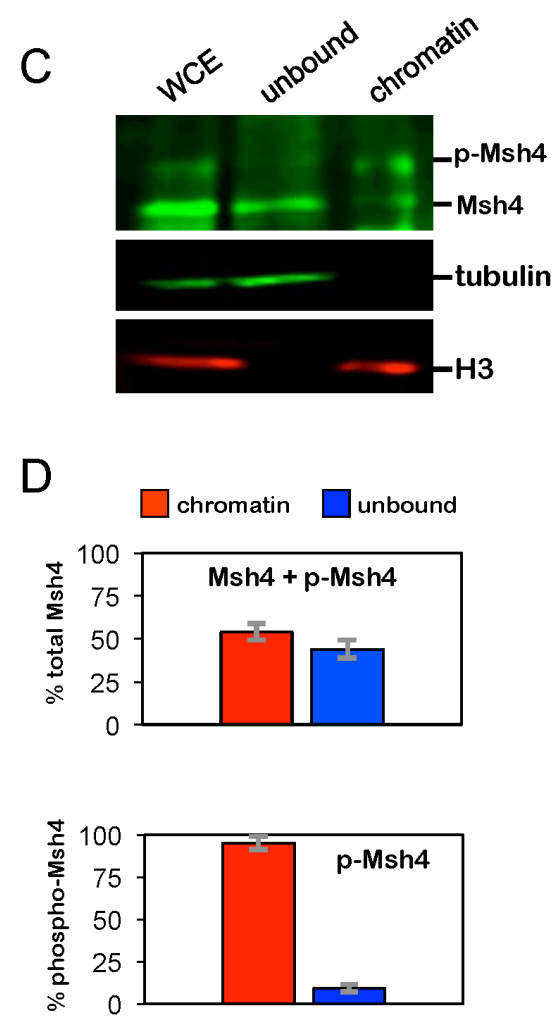

Figure 6. Genetic requirements and chromatin association of phosphorylated of Msh4.

(A) Chromosomal and recombination events of meiosis illustrating the steps affected by mutants analyzed in panel B. Blue, chromatin; red lines, homolog axes; yellow line, synaptonemal complex central region. 
bioRxiv preprint doi: https://doi.org/10.1101/386458; this version posted August 27, 2018. The copyright holder for this preprint (which was not certified by peer review) is the author/funder, who has granted bioRxiv a license to display the preprint in perpetuity. It is made available under aCC-BY-NC-ND 4.0 International license.

(B) Western analysis of Msh4 in the indicated mutants.

(C) Western analysis of Msh4 from whole cell extracts ("WCE”) and extracts separated into soluble ("unbound") and chromatin fractions; tubulin and histone H3 were used as markers for these two fractions, respectively. (D) Quantification of total Msh4 (top graph) and phosphorylated Msh4 (bottom graph) in the two fractions. Means values $\pm S$.E. were calculated from three independent experiments. 


\section{A}

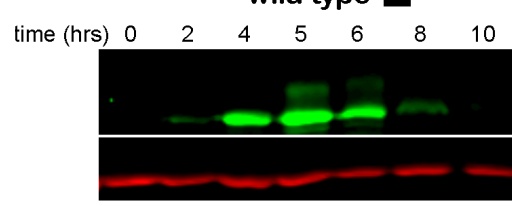

$P_{C L B 2}-M E C 1$
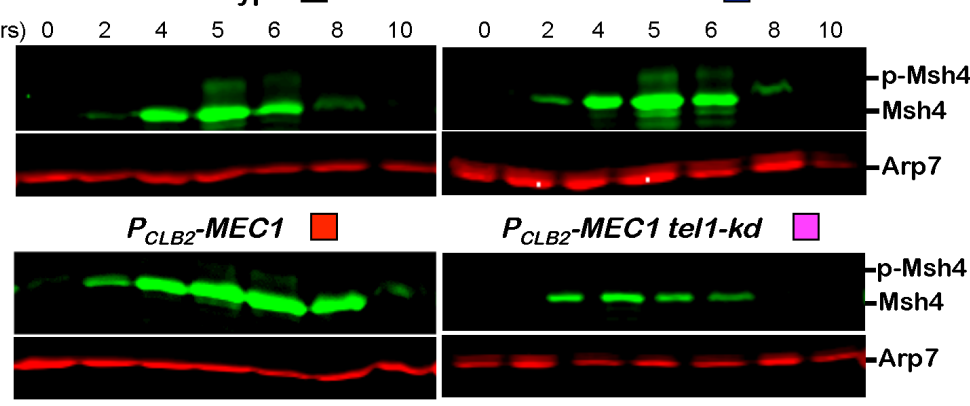

$P_{C L B 2}-M E C 1$ tel1-kd $\square$

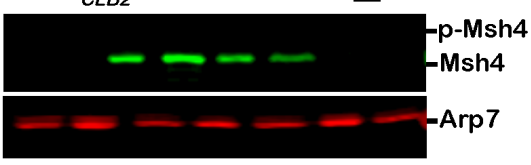

$$
\text { C }
$$

$\operatorname{mek1} \Delta$

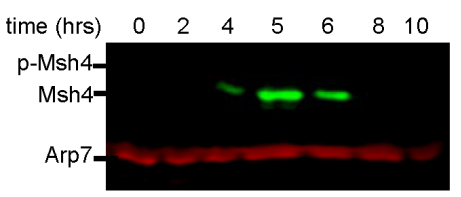

$\mathrm{F}$

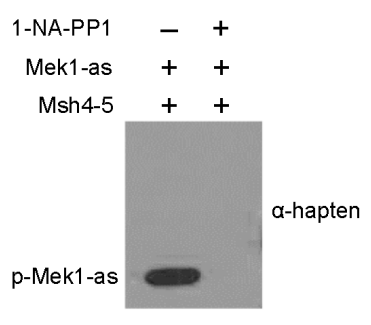

G

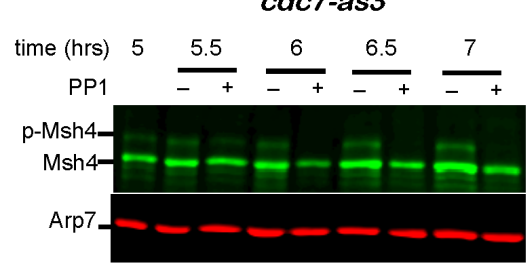

B

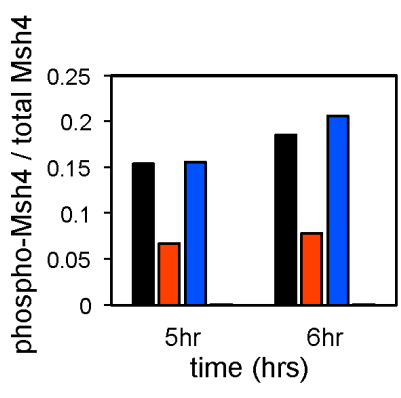

E

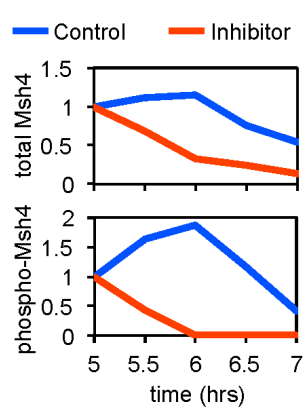

$\mathrm{H}-$ Control - Inhibitor
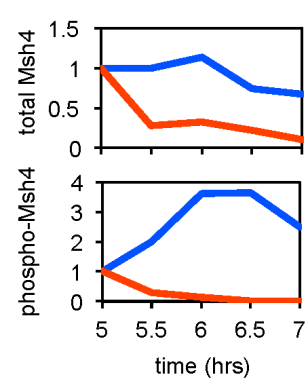

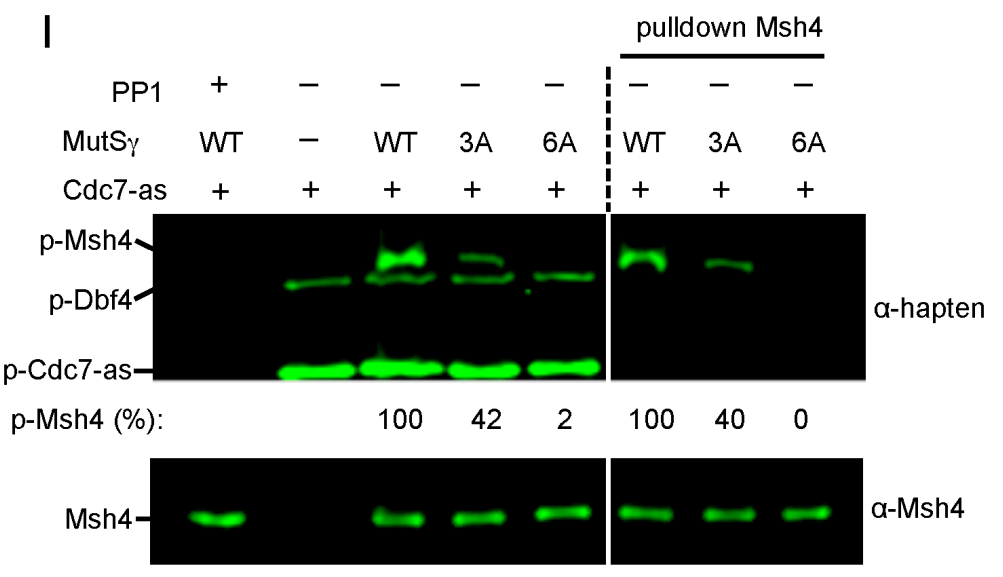

Figure 7. Kinase requirements for phosphorylation of Msh4 in vivo and in vitro.

(A) Western analysis of Msh4 in tel1-kd, pCLB2-MEC1 and pCLB2-MEC1 tel1-kd strains during meiosis. 
(B) Fraction of Msh4 that is phosphorylated at 5 and 6 hrs in the experiments shown in panel $(A)$.

(C) Western analysis of Msh4 in a mek1 $\Delta$ null mutant.

(D) Western analysis of Msh4 in strains containing the ATP-analog sensitive mek1-as allele, with and without addition of the inhibitor 1-NA-PP1 at $5 \mathrm{hrs}$.

(E) Relative levels of total and phosphorylated Msh4 quantified from the experiment shown in panel (D). Levels were normalized to the $5 \mathrm{hr}$ time point.

(F) Western analysis of a Mek1-as (GST-Mek1-as) in vitro kinase assay with MutS $\gamma$, with and without the inhibitor 1-NA-PP1.

(G) Western analysis of Msh4 in strains containing the ATP-analog sensitive cdc7-as allele, with and without addition of the inhibitor PP1 at $5 \mathrm{hrs}$. The anti-thiophosphate ester ( $\alpha$-hapten) antibody recognizes phosphorylation products of the semi-synthetic epitope system.

(H) Relative levels of total and phosphorylated Msh4 quantified from the experiment shown in panel $(G)$. Levels were normalized to the 5 hrs timepoint.

(I) Western analysis of an in vitro kinase assay with the Cdc7-as3 kinase (FLAG-Cdc7-as3Dbf4 complex) with wild-type MutS $\gamma$ or mutant derivatives containing serine-alanine substitutions in the N-terminus of Msh4. In the right-hand panel, Msh4 was purified from the reactions.

Phosphorylation efficiency was calculated relative to wild-type Msh4. In the lower panels, 10\% of each reaction was probed for Msh4 as a loading control. Also see Figure S6. 